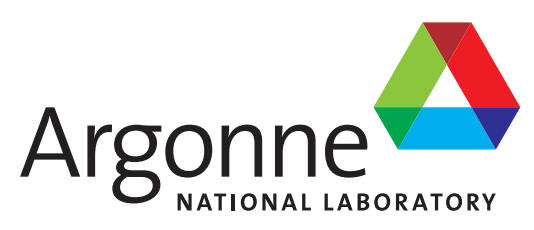

ANL/MCS-TM-320

\title{
Numerical Homogenization on Approach for Stokesian Suspensions
}

Mathematics and Computer Science Division 
About Argonne National Laboratory

Argonne is a U.S. Department of Energy laboratory managed by UChicago Argonne, LLC under contract DE-AC02-06CH11357. The Laboratory's main facility is outside Chicago, at 9700 South Cass Avenue, Argonne, Illinois 60439. For information about Argonne and its pioneering science and technology programs, see www.anl.gov.

\section{Availability of This Report}

This report is available, at no cost, at http://www.osti.gov/bridge. It is also available on paper to the U.S. Department of Energy and its contractors, for a processing fee, from:

U.S. Department of Energy

Office of Scientific and Technical Information

P.O. Box 62

Oak Ridge, TN 37831-0062

phone (865) 576-8401

fax (865) 576-5728

reports@adonis.osti.gov

\section{Disclaimer}

This report was prepared as an account of work sponsored by an agency of the United States Government. Neither the United States Government nor any agency thereof, nor UChicago Argonne, LLC, nor any of their employees or officers, makes any warranty, express or implied, or assumes any legal liability or responsibility for the accuracy, completeness, or usefulness of any information, apparatus, product, or process disclosed, or represents that its use would not infringe privately owned rights. Reference herein to any specific commercial product, process, or service by trade name, trademark, manufacturer, or otherwise, does not necessarily constitute or imply its endorsement, recommendation, or favoring by the United States Government or any agency thereof. The views and opinions of document authors expressed herein do not necessarily state or reflect those of the United States Government or any agency thereof, Argonne National Laboratory, or UChicago Argonne, LLC. 


\section{Numerical Homogenization on Approach for Stokesian Suspensions}

by

Brian M. Haines, ${ }^{1,2}$ Leonid V. Berlyand, ${ }^{2}$ and Dmitry A. Karpeev ${ }^{1}$

${ }^{1}$ Mathematics and Computer Science Division, Argonne National Laboratory

${ }^{2}$ Department of Mathematics, Pennsylvania State University

November 10, 2011 


\section{Contents}

1 Introduction 1

2 Bacterial suspensions 1

2.1 Mathematical model . . . . . . . . . . . . . . . . . . . . . . . . . 2

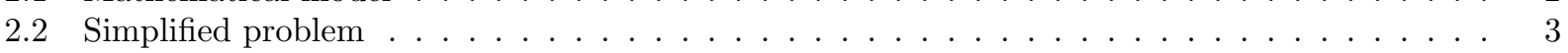

3 Spectral basis 3

4 Advection of the basis 5

4.1 Perturbation of Stokes eigenvalues . . . . . . . . . . . . . . . . . . . . . 5

4.2 Error estimate for advected basis . . . . . . . . . . . . . . . . . . . . . . . . . . . . . 9

4.3 Full error estimate . . . . . . . . . . . . . . . . . . . . . . . . . 13

5 Basis localization 14

5.1 Stokes Neumann eigenfunctions . . . . . . . . . . . . . . . . . . . . . . 14

5.2 Localized basis . . . . . . . . . . . . . . . . . . . . . . . . . . . 15

6 Conclusion 22

Acknowledgments 22

References 


\section{Introduction}

In this technical report we investigate efficient methods for numerical simulation of active suspensions. The prototypical system is a suspension of swimming bacteria in a Newtonian fluid. Rheological and other macroscopic properties of such suspensions can differ dramatically from the same properties of the suspending fluid alone or of suspensions of similar but inactive particles [2,4,5,7,10-12,15-22,26,27,30,31,33-35,38-42]. Elongated bacteria, such as E. coli or B. subtilis, swim along their principal axis, propelling themselves with the help of flagella, attached at the anterior of the organism and pushing it forward in the manner of a propeller. They interact hydrodynamically with the surrounding fluid and, because of their asymmetrical shape, have the propensity to align with the local flow. This, along with the dipolar nature of bacteria (the two forces a bacterium exerts on a fluid - one due to self-propulsion and the other opposing drag - have equal magnitude and point in opposite directions), causes nearby bacteria to tend to align, resulting in a intermittent local ordering on the mesoscopic scale, which is between the microscopic scale of an individual bacterium and the macroscopic scale of the suspension (e.g., its container).

The local ordering is sometimes called a collective mode or collective swimming. Thanks to self-propulsion, collective modes inject momentum into the fluid in a coherent way. This enhances the local strain rate without changing the macroscopic stress applied at the boundary of the container. The macroscopic effective viscosity of the suspension is defined roughly as the ratio of the applied stress to the bulk strain rate. If local alignment and therefore local strain-rate enhancement, are significant, the effective viscosity can be appreciably lower than that of the corresponding passive suspension or even of the surrounding fluid alone (see, e.g., $[10-12,30,31]$ ). Indeed, a sevenfold decrease in the effective viscosity was observed in experiments with $B$. subtilis [33]. More generally, local collective swimming resulting from bacterial alignment can significantly alter other macroscopic properties of the suspension, such as the oxygen diffusivity [38] and mixing rates $[19,35]$.

In order to understand the unique macroscopic properties of active suspensions the connection between microscopic swimming and alignment dynamics and the mesoscopic pattern formation must be clarified. This is difficult to do analytically in the fully general setting of moderately dense suspensions, because of the large number of bacteria involved (approx. $10^{10} \mathrm{~cm}^{-3}$ in experiments) and the complex, time-dependent geometry of the system. Many reduced analytical models of bacterial have been proposed $[13,25,28,32]$, but all of them require validation. While comparison with experiment is the ultimate test of a model's fidelity, it is difficult to conduct experiments matched to these models' assumptions. Numerical simulation of the microscopic dynamics is an acceptable substitute, but it runs into the problem of having to discretize the fluid domain with a fine-grained boundary (the bacteria) and update the discretization as the domain evolves (bacteria move). This leads to a prohibitively high number of degrees of freedom and prohibitively high setup costs per timestep of simulation.

In this technical report we propose numerical methods designed to alleviate these two difficulties. We indicate how to (1) construct an optimal discretization in terms of the number of degrees of freedom per digit of accuracy and (2) optimally update the discretization as the simulation evolves. The technical tool here is the derivation of rigorous error bounds on the error in the numerical solution when using our proposed discretization at the initial time as well as after a given elapsed simulation time. These error bounds should guide the construction of practical discretization schemes and update strategies. Our initial construction is carried out by using a theoretically convenient, but practically prohibitive spectral basis, which is a Galerkin basis of functions with global support. At the end of this report we propose localization techniques while maintaining acceptable error bounds. No numerical experiments were conducted as part of this study, but we envision that we may undertake such studies and further development of the method, jointly or individually.

\section{Bacterial suspensions}

We use a model of a bacterial suspension in the simplest way that permits our analysis and captures its two important qualities: self-propulsion and a propensity of the bacteria to align with the local ambient flow. Alignment is chiefly due to an elongated shape of the bacteria, such as that of B. subtilis (see, e.g., Fig. 1) 
used in experiments [33]. For our analysis the shape is essentially immaterial, so we assume that the $l$-th bacterium is represented by a smooth convex body $B^{l}$, which for concreteness can be taken to be a prolate spheroid.

Propulsion can take many different forms, from multiple flagella distributed over a bacterium's body, which tend to bundle together when rotating and apply the thrust primarily behind one of the ends (the "tail") of an elongated body, to a pair of flagella executing a "breaststroke" at the "head" of the organism (such as Chlamydomonas, which is an alga, not bacterium, but is similar from our point of view), to cilia distributed over all or a portion of a bacterium's body and beating more or less independently. Several models of propulsion have been proposed in the literature $[11,14,16,32]$. They essentially prescribe a body force in the fluid and away from $B^{l}$, representing the action of the flagella, or a boundary condition prescribing the fluid velocity on a part of the organism's boundary $\Gamma_{1}^{l} \subset \partial B^{l}$ and tangential tractions on another $\Gamma_{2}^{l} \subset \partial B^{l}$. The body force can be singular, such as a delta force applied at a fixed position and orientation relative to the body coordinates of the bacterium. This could be viewed as an idealized model of flagellar action concentrated on a very small portion of the fluid. Whenever a boundary condition model of self-propulsion is used, prescribed tangential tractions have to be supplemented with a vanishing of the normal component of the velocity on $\Gamma_{2}^{l}$ (reflecting the fact that fluid cannot penetrate the bacteria's bodies), and the complement $\partial B^{l} \backslash\left(\Gamma_{1}^{l} \cup \Gamma_{2}^{l}\right)$ must be supplied with the no-slip boundary conditions.

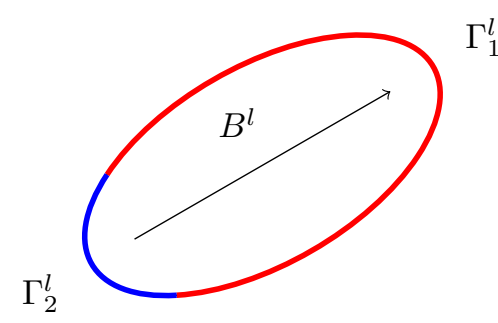

Figure 1: A two-dimensional illustration of a prolate spheroidal model of a swimmer. Shown are the velocity $\left(\Gamma_{1}^{l}\right.$, red) and traction $\left(\Gamma_{2}^{l}\right.$, blue) portions of the boundary and the orientation vector.

In our analysis the precise form of the propulsion model is not important. We start with a general model that includes a body force and the two types of boundary conditions, with the no-slip part absorbed into $\Gamma_{1}^{l}$ so that $\Gamma_{1}^{l} \cup \Gamma_{2}^{l}=\partial B^{l}$. Since the precise biological nature of the swimming organism (bacterium, alga, microswimmer) is immaterial for our study, we will use these terms interchangeably, occasionally substituting "particle" or "inclusion" for them, as well. With these remarks, we can state the mathematical model of a bacterial suspension. After that we indicate how to reduce this model to a simplified problem, which we then analyze. The analysis starts with the description of an approximation (discretization), based on the Stokes spectral basis. We show the optimality of the resulting initial $H^{1}$ approximation error and how to evolve this basis as the bacteria move while maintaining error control. Since the spectral basis is global and therefore of little practical use in numerical applications, we discuss approaches to the localization of the basis and the impact of basis localization on the obtained error estimates.

\subsection{Mathematical model}

We model a suspension of $N$ neutrally buoyant (active or passive) particles using the Stokes equation with mixed boundary conditions:

$$
\left\{\begin{array}{lr}
-\eta \Delta u+\nabla p=f, \nabla \cdot u=0 & x \in V_{F}:=V \backslash \cup_{l} B^{l} \\
u=v^{l}+\omega^{l} \times\left(x-x^{l}\right) & x \in \Gamma_{1} \cap \partial B^{l} \\
u \cdot n=\left[v^{l}+\omega^{l} \times\left(x-x^{l}\right)\right] \cdot n & x \in \Gamma_{2} \cap \partial B^{l} \\
\sigma(u) n-n(\sigma(u): n n)=g & x \in \Gamma_{2} \cap \partial B^{l} \\
u=h & x \in \partial V \\
\frac{d x}{d t}=u & x \in \Gamma_{1} \cup \Gamma_{2} \\
\int_{\partial B^{l}} \sigma(u) n d S+F^{l}=0, \int_{\partial B^{l}} \sigma(u) n \times\left(x-x^{l}\right) d S+T^{l}=0, &
\end{array}\right.
$$

where $V \subset \mathbb{R}^{d}, d \geq 2$, is such that $\partial V$ is smooth (e.g., $C^{\infty}$ ), $\Gamma_{1} \cap \Gamma_{2}=\emptyset, \Gamma_{1} \cup \Gamma_{2}=\cup_{l} \partial B^{l}, x^{l}$ is the center of $B^{l}$, defined as

$$
x^{l}:=\frac{1}{\left|B^{l}\right|} \int_{B^{l}} x d x
$$


$\sigma(u):=-p I+2 \eta e(u)$ is the stress tensor, $e(u):=\frac{1}{2}\left(\nabla u+\nabla u^{T}\right)$ is the rate of strain, $f \in H^{(2 d-1) / 2}\left(V_{F}\right)$, $h \in H^{d}(\partial V)$, and $F^{l}, T^{l} \in \mathbb{R}^{3}$. We also require that $\int_{\partial V} h \cdot n d S=0$ so that we may have $\nabla \cdot u=0$. All quantities, including the domain, are time dependent via the balance of forces and torques. The existence and uniqueness of solutions to equation (2.1) are proved in [9].

\subsection{Simplified problem}

The problem can be simplified by considering the solution of

$$
\left\{\begin{array}{lr}
-\eta \Delta u+\nabla p=f, \nabla \cdot u=0 & x \in V_{F}:=V \backslash \cup_{l} B^{l} \\
u=0 & x \in \Gamma_{1} \cap \partial B^{l} \\
u=0 & x \in \partial V \\
u \cdot n=0 & x \in \Gamma_{2} \cap \partial B^{l} \\
\sigma(u) n-n(\sigma(u): n n)=0 & x \in \Gamma_{2} \cap \partial B^{l} \\
\frac{d x}{d t}=v & x \in \partial V,
\end{array}\right.
$$

where $v(x, t)$ is sufficiently regular (as we will show below, $\left(C^{3}\left(V_{F}\right)\right)^{d}$ is sufficient) for each $t \in[0, T]$ subject to $\nabla \cdot v=0$. We can use equation (2.2) to solve equation (2.1) by first constructing divergence-free functions $\zeta^{l}$ with disjoint support ${ }^{1}$ whose sum satisfies the boundary conditions in equation (2.1). One can then produce a solution to equation (2.1) by adding a corrector, which is a solution to equation (2.2) with $f=\sum_{l} \zeta^{l}$.

\section{Spectral basis}

For simplicity, we will henceforth consider purely Dirichlet boundary conditions (that is, $\Gamma_{2}=\emptyset$ ). Our construction readily applies to the general case that includes Neumann boundary conditions by considering the corresponding eigenfunctions. For a fixed time $t \in[0, \infty)$, let $S(t)$ denote the closure of the set

$$
\left\{v \in\left(C^{\infty}\left(V_{F}(t)\right)\right)^{d} \mid \nabla \cdot v=0, v=0 \text { for } x \in \partial V_{F}(t)\right\}
$$

in the $\left(L^{2}\left(V_{F}(t)\right)\right)^{d}$ norm, and let $S^{m}(t):=S(t) \cap\left(H^{m}\left(V_{F}(t)\right)\right)^{d} . S(t)$ is equivalently defined [36] as

$$
S(t):=\left\{v \in\left(L^{2}\left(V_{F}(t)\right)\right)^{d} \mid \nabla \cdot v=0, v \cdot n=0 \text { for } x \in \partial V_{F}(t)\right\},
$$

where $\nabla \cdot v=0$ is to be understood in a weak sense and the equality on the boundary must be understood in the sense of traces in $\left(H^{-1 / 2}\left(\partial V_{F}(t)\right)\right)$. Furthermore, we can decompose $\left(L^{2}\left(V_{F}(t)\right)\right)^{d}=S(t) \oplus S^{\perp}(t)$, where

$$
S^{\perp}(t):=\left\{\nabla p \mid p \in H^{1}\left(V_{F}(t)\right)\right\} .
$$

The following theorem is proved in, for example, [36].

Theorem 3.1. Let $t \in(0, \infty)$ be fixed. For all $f \in S^{m}(t)$ and $m \in \mathbb{N}$, the equation

$$
\begin{cases}-\eta \Delta u+\nabla p=f, \nabla \cdot u=0 & x \in V_{F}(t) \\ u=0 & x \in V_{F}(t)\end{cases}
$$

has a unique solution $u \in S^{m+2}(t)$ with $p \in H^{m+1}\left(V_{F}(t)\right)$. Furthermore, $\exists C_{m}>0$ such that $(u, p)$ satisfies

$$
\|u\|_{\left(H^{m+2}\left(V_{F}(t)\right)\right)^{d}}+\|p\|_{H^{m+1}\left(V_{F}(t)\right)} \leq C_{m}\|f\|_{\left.H^{m}\left(V_{F}(t)\right)\right)^{d}} .
$$

It is a standard result that $S(t)$ is compactly embedded in $S^{2}(t)$. Therefore, the solution operator $\mathcal{S}^{-1}(t): S(t) \rightarrow S(t) \supset S^{2}(t)$ to the Stokes equation is compact. Hence, the Dirichlet eigenfunctions of the Stokes equation form a complete basis for $S(t)$. Furthermore, the set of eigenvalues $\left\{\lambda_{k}\right\}$ is countable, and $\lambda_{k}>0$ for all $k$.

\footnotetext{
${ }^{1}$ Giving the functions disjoint support allows one to enforce the constraints (balance of forces and torques) in equation (2.1).
} 
Let $V^{h}$ denote the span of the first $M$ Stokes eigenfunctions $\left(\psi_{t}^{k}, \phi_{t}^{k}\right)$ (ordered such that their eigenvalues are increasing). In particular, $\left(\psi_{t}^{k}, \phi_{t}^{k}\right)$ satisfy

$$
\left\{\begin{array}{lr}
-\eta \Delta \psi_{t}^{k}+\nabla \phi_{t}^{k}=\lambda_{t}^{k} \psi_{t}^{k}, \nabla \cdot \psi_{t}^{k}=0 & x \in V_{F}(t) \\
\psi_{t}^{k}=0 & x \in \partial V_{F}(t)
\end{array}\right.
$$

Without loss of generality, we will assume that the eigenvalues are ordered such that $0<\lambda_{1} \leq \lambda_{2} \leq \ldots$. Methods for solving Stokes eigenvalue problems numerically are outlined in, for example, [6,23]. Let $(f, g)_{t}$ denote the $L^{2}\left(V_{F}(t)\right)$ inner product of $f$ and $g$ and

$$
\begin{aligned}
u(t) & =\sum_{k=1}^{\infty} c_{k}(t) \psi_{t}^{k}, \\
u_{h}(t) & =\sum_{k=1}^{M} c_{k}(t) \psi_{t}^{k},
\end{aligned}
$$

where $c_{k}(t):=\left(u(t), \psi_{t}^{k}\right)_{t}$. We can estimate the finite-element method (FEM) error as

$$
\begin{aligned}
\left\|u(t)-u_{h}(t)\right\|_{L^{2}\left(V_{F}(t)\right)}^{2} & =\sum_{k=M+1}^{\infty} c_{k}^{2}(t)=\sum_{k=M+1}^{\infty} \frac{\left(f, \psi_{t}^{l}\right)_{t}^{2}}{\left(\lambda_{t}^{l}\right)^{2}} \\
& \leq \frac{1}{\left(\lambda_{t}^{M+1}\right)^{2}}\|f\|_{L^{2}\left(V_{F}(t)\right)}^{2}
\end{aligned}
$$

One can also estimate the $H^{1}$-seminorm error as

$$
\begin{aligned}
\left\|\nabla u(t)-\nabla u_{h}(t)\right\|_{L^{2}\left(V_{F}(t)\right)}^{2} & =\sum_{k=M+1}^{\infty} \lambda_{t}^{k} c_{k}^{2}(t)=\sum_{k=M+1}^{\infty} \lambda_{t}^{k} \frac{\left(f, \psi_{t}^{k}\right)^{2}}{\left(\lambda_{t}^{k}\right)^{2}} \\
& \leq \frac{1}{\lambda_{t}^{M+1}}\|f\|_{L^{2}\left(V_{F}(t)\right)}^{2}
\end{aligned}
$$

For Dirichlet boundary conditions, the eigenvalues of the Stokes equation have the asymptotic behavior (see, e.g., [1] $(d=3),[24](d \geq 2))$

$$
\lambda_{t}^{k} \sim \eta\left(\frac{(2 \pi)^{d}}{\omega_{d}(d-1)}\right)^{\frac{2}{d}}\left|V_{F}\right|^{-2 / d} k^{2 / d}
$$

where $\omega_{d}$ is the volume of the unit ball in $\mathbb{R}^{d}$. Therefore, if we set $M=h^{-d}\left|V_{F}\right|$, that is, the number of degrees of freedom in a piecewise linear basis on a triangulation of $V_{F}$, we can rewrite equations (3.3) and (3.4) as

$$
\lim _{h \rightarrow 0} \frac{\left\|u-u_{h}\right\|_{L^{2}(V)}}{h^{2}\|f\|_{L^{2}(V)}}=\frac{1}{\eta}\left(\frac{\omega_{d}(d-1)}{(2 \pi)^{d}}\right)^{\frac{2}{d}}
$$

and

$$
\lim _{h \rightarrow 0} \frac{\left\|\nabla u-\nabla u_{h}\right\|_{L^{2}(V)}}{h\|f\|_{L^{2}(V)}}=\frac{1}{\sqrt{\eta}}\left(\frac{\omega_{d}(d-1)}{(2 \pi)^{d}}\right)^{\frac{1}{d}},
$$

respectively.

To resolve the particle boundaries, one can represent the basis functions using a triangulation in a Lagrangian coordinate system or using an arbitrary Lagrangian-Eulerian method. 


\section{Advection of the basis}

To produce an efficient method, we wish to avoid recomputing the basis of $V^{h}$ at every timestep $t_{i}:=i \tau$, where $\tau>0$. Henceforth, we will allow the domain $V_{F}$ to evolve, and we will denote by $V_{F}(t)$ the domain at time $t$. Instead of recomputing the basis at each discrete timestep, we will assume that at each timestep we are given a vector field $v_{t_{i}} \in C^{\infty}\left(V_{F}\left(t_{i}\right)\right)$, which we will use to advect the domain and basis. For $x \in V_{F}(t)$, let $F_{t_{i+1}}(x):=x+v_{t_{i}}(x) \tau$. We will define $V_{F}\left(t_{i+1}\right)=\left\{F(x) \mid x \in V_{F}\left(t_{i}\right)\right\}$. The advected basis functions will be referred to by $A_{t_{i}} \psi_{0}^{k}$, where $\psi_{0}^{k}$ is the Stokes eigenfunction in $V_{F}(0)$ corresponding to the eigenvalue $\lambda_{0}^{k}$. In the context of solving equation (2.1), this will simply be the FEM solution of (2.1) at the previous timestep - that is, $v_{t_{i}}=u_{h}\left(t_{i-1}\right)$. The basis functions will be advected by defining

$$
A_{t_{i+1}} \psi_{0}^{k}(x):=\nabla F_{t_{i+1}}\left(F_{t_{i+1}}^{-1}(x)\right) A_{t_{i}} \psi_{0}^{k}\left(F_{t_{i+1}}^{-1}(x)\right) .
$$

Note that for $\tau \rightarrow 0, A_{t_{i+1}} \psi_{0}^{k}$ is the Taylor expansion of the solution to

$$
\left\{\begin{array}{lr}
\frac{\partial A_{t_{i+1}} \psi_{0}^{k}}{\partial t}+\left[v_{t_{i}}, A_{t_{i+1}} \psi_{0}^{k}\right]=0 & x \in V_{F}\left(t_{i+1}\right), \\
A_{t_{i+1}} \psi_{0}^{k}=0 & x \in \partial V_{F}\left(t_{i+1}\right)
\end{array}\right.
$$

where $[\xi, \chi]$ is the Lie bracket defined by

$$
[\xi, \chi]:=\xi \frac{\partial \chi}{\partial x}-\chi \frac{\partial \xi}{\partial x}
$$

Furthermore, $A_{t_{i}} \psi_{0}^{k}$ is divergence free for all $i$. Nevertheless, $A_{\tau} \psi_{0}^{k}$ does not produce a good approximation to $\psi_{\tau}^{k}$-in particular, the advection in equation (4.2) does not preserve the $L^{2}$ norm of $\psi_{0}^{k}$. Therefore, we construct a "corrector" in order to create a good approximation. We can do this by solving the system

$$
\mathcal{A}_{t_{i+1}} \psi_{0}^{k}(x):=A_{\tau} \mathcal{A}_{t_{i}} \psi_{0}^{k}(x)+\tau \sum_{j=1}^{M} a_{j}^{k} \mathcal{A}_{t_{i+1}} \psi_{0}^{j}(x),
$$

where, for $j \neq k$,

$$
\begin{aligned}
a_{j}^{k}= & \frac{\eta}{\lambda_{t_{i}}^{j}-\lambda_{t_{i}}^{k}}\left[2 \int_{V_{F}\left(t_{i}\right)}\left(\nabla \mathcal{A}_{t_{i}} \psi_{0}^{j} e\left(v_{t_{i}}\right)\right): \nabla \mathcal{A}_{t_{i}} \psi_{0}^{k} d x\right. \\
& +\int_{V_{F}\left(t_{i}\right)}\left[\left(\nabla^{2} v_{t_{i}} \nabla \mathcal{A}_{t_{i}} \psi_{0}^{j}\right) \cdot \mathcal{A}_{t_{i}} \psi_{0}^{k}-2\left(\nabla \mathcal{A}_{t_{i}} \psi_{0}^{k} \nabla^{2} v_{t_{i}}\right) \cdot \mathcal{A}_{t_{i}} \psi_{0}^{j}\right] d x \\
& -\int_{V_{F}\left(t_{i}\right)}\left[e\left(v_{t_{i}}\right):\left(\nabla \mathcal{A}_{t_{i}} \psi_{0}^{j}\left(\nabla \mathcal{A}_{t_{i}} \psi_{0}^{k}\right)^{T}\right)+\left(\mathcal{A}_{t_{i}} \psi_{0}^{j} \nabla^{2} v_{t_{i}}\right): \nabla \mathcal{A}_{t_{i}} \psi_{0}^{k}\right] d x \\
& \left.+2 \lambda_{t_{i}}^{k} \int_{V_{F}\left(t_{i}\right)} \mathcal{A}_{t_{i}} \psi_{0}^{k} \mathcal{A}_{t_{i}} \psi_{0}^{j}: e\left(v_{t_{i}}\right) d x\right]
\end{aligned}
$$

and, for $j=k$,

$$
a_{k}^{k}=-\int_{V_{F}\left(t_{i}\right)}\left(\mathcal{A}_{t_{i}} \psi_{0}^{k} e\left(v_{t_{i}}\right)\right) \cdot \mathcal{A}_{t_{i}} \psi_{0}^{k} d x .
$$

The derivation of these formulae is left for the proof of Theorem 4.3.

\subsection{Perturbation of Stokes eigenvalues}

To study the error in advecting the basis, we will need to understand how the eigenvalues of the Stokes equation change upon perturbing the domain. We will first consider the case of Dirichlet boundary conditions. 
Let $\left(\psi_{t}^{k}, \phi_{t}^{k}\right)$ be the solution of

$$
\left\{\begin{array}{lr}
-\eta \Delta \psi_{t}^{k}+\nabla \phi_{t}^{k}=\lambda_{t}^{k} \psi_{t}^{k}, \nabla \cdot \psi_{t}^{k}=0 & x \in V_{F}(t) \\
\psi=0 & x \in \partial V_{F}(t)
\end{array}\right.
$$

where $V_{F}$ is a bounded domain with a smooth $\left(C^{\infty}\right)$ time-dependent boundary. The velocity of the boundary $\nu$ is defined by the limit $[8]$

$$
\nu:=\lim _{t \rightarrow 0} \frac{(x(t)-x(0)) \cdot n(0)}{t},
$$

where $x(0) \in \partial V_{F}(0), x(t) \in \partial V_{F}(t)$, the line between $x(0)$ and $x(t)$ is perpendicular to $\partial V_{F}(0)$ at $x(0)$, and $n(0)$ is the normal to $\partial V_{F}(0)$. Note that in equation (2.2), we have $\nu=v \cdot n$.

The rate of change of an eigenvalue is defined by

$$
\lambda_{t}^{k, \prime}:=\frac{d \lambda_{t}^{k}}{d t}
$$

We will use the moving equivalent of the fundamental theorem of calculus, given by (see, e.g., [8])

$$
\frac{d}{d t} \int_{V_{F}(t)} F d x=\int_{V_{F}(t)} \frac{\partial F}{\partial t} d x+\int_{\partial V_{F}(t)} \nu F d S
$$

and the corresponding formula for surfaces

$$
\frac{d}{d t} \int_{\partial V_{F}(t)} F d S=\int_{\partial V_{F}(t)} \frac{\delta F}{\delta t} d S-\int_{\partial V_{F}(t)} \nu \kappa F d S
$$

where $\kappa$ is the mean curvature of $\partial V_{F}$ and $\frac{\delta F}{\delta t}$ is a derivative defined for scalar fields on moving surfaces, given by

$$
\frac{\delta F}{\delta t}:=\frac{\partial F}{\partial t}+\nu n \cdot \nabla F
$$

Theorem 4.1. The rate of change $\lambda^{k, \prime}$ of a simple eigenvalue $\lambda_{t}^{k}$ of equation (4.5) is given by

$$
\lambda^{k, \prime}=-\eta \int_{\partial V_{F}(t)} \nu \nabla \psi_{t}^{k}: \nabla \psi_{t}^{k} d S
$$

where $\nu$ is given in equation (4.6).

Remark 4.1. This proof is similar to the proof of Hadamard's formula given in [8].

Proof. First, differentiating equation (4.5) and applying equation (4.10) to the boundary conditions, note that $\frac{\partial \psi}{\partial t}$ solves

$$
\left\{\begin{array}{lr}
-\eta \Delta \frac{\partial \psi_{t}^{k}}{\partial t}+\nabla \frac{\partial \phi_{t}^{k}}{\partial t}=\lambda_{t}^{k, \prime} \psi_{t}^{k}+\lambda_{t}^{k} \frac{\partial \psi_{t}^{k}}{\partial t}, \nabla \cdot \frac{\partial \psi_{t}^{k}}{\partial t}=0 & x \in V_{F}(t) \\
\frac{\partial \psi_{t}^{k}}{\partial t}=-\nu \frac{\partial \psi_{t}^{k}}{\partial n} & x \in \partial V_{F}(t) .
\end{array}\right.
$$

Multiplying equation (4.5) by $\psi_{t}^{k}$ and integrating by parts, we can write lambda as

$$
\lambda_{t}^{k}=\eta \int_{V_{F}(t)} \nabla \psi_{t}^{k}: \nabla \psi_{t}^{k} d x
$$

Therefore, using equation (4.8), we have

$$
\begin{aligned}
\lambda_{t}^{k, \prime} & =\eta \frac{d}{d t} \int_{V_{F}(t)} \nabla \psi_{t}^{k}: \nabla \psi_{t}^{k} d x \\
& =2 \eta \int_{V_{F}(t)} \nabla \psi_{t}^{k}: \nabla \frac{\partial \psi_{t}^{k}}{\partial t} d x+\eta \int_{\partial V_{F}(t)} \nu \nabla \psi_{t}^{k}: \nabla \psi_{t}^{k} d S .
\end{aligned}
$$


Integrating by parts, we get

$$
=-2 \eta \int_{V_{F}(t)} \psi_{t}^{k} \cdot \Delta \frac{\partial \psi_{t}^{k}}{\partial t} d x+\eta \int_{\partial V_{F}(t)} \nu \nabla \psi_{t}^{k}: \nabla \psi_{t}^{k} d S .
$$

From equation (4.12), this is equal to

$$
=2 \int_{V_{F}(t)} \psi_{t}^{k} \cdot\left(\lambda^{k, \prime} \psi_{t}^{k}+\lambda_{t}^{k} \frac{\partial \psi_{t}^{k}}{\partial t}-\nabla \frac{\partial \phi_{t}^{k}}{\partial t}\right) d x+\eta \int_{\partial V_{F}(t)} \nu \nabla \psi_{t}^{k}: \nabla \psi_{t}^{k} d S .
$$

Note that

$$
\int_{V_{F}(t)} \psi_{t}^{k} \cdot \psi_{t}^{k} d x=1
$$

and hence

$$
\frac{d}{d t} \int_{V_{F}(t)} \psi_{t}^{k} \cdot \psi_{t}^{k} d x=2 \int_{V_{F}(t)} \psi_{t}^{k} \cdot \frac{\partial \psi_{t}^{k}}{\partial t} d x=0,
$$

since $\psi_{t}^{k}=0$ on $\partial V_{F}(t)$. Furthermore, integrating by parts, we obtain

$$
\int_{V_{F}(t)} \psi_{t}^{k} \cdot \nabla \frac{\partial \phi_{t}^{k}}{\partial t} d x=\int_{V_{F}(t)} \nabla \cdot \psi_{t}^{k} \frac{\partial \phi_{t}^{k}}{\partial t} d x+\int_{\partial V_{F}(t)} \psi_{t}^{k} \frac{\partial \phi_{t}^{k}}{\partial t} \cdot n d S=0,
$$

from equation (4.5). Combining equations (4.14)-(4.17) yields equation (4.11).

The following lemma will be needed to determine $\lambda^{\prime}$ for eigenvalues that are not simple.

Lemma 4.1. For all $i, k \in \mathbb{N}$,

$$
\int_{\partial V_{F}(t)}\left(v \nabla \psi_{t}^{k}\right) \cdot n \phi_{t}^{i} d S=0
$$

Proof. Using the divergence theorem and the boundary conditions on $\psi_{t}^{k}$, we have

$$
0=\int_{\partial V_{F}(t)} \psi_{t}^{k} \cdot n \phi_{t}^{i} d S=\int_{V_{F}(t)} \psi_{t}^{k} \cdot \nabla \phi_{t}^{i} d x
$$

Therefore, using equation (4.8) and the boundary conditions on $\psi_{t}^{k}$, we get

$$
\begin{aligned}
0 & =\frac{d}{d t} \int_{V_{F}(t)} \psi_{t}^{k} \cdot \nabla \phi_{t}^{i} d x \\
& =\int_{V_{F}(t)}\left[\frac{\partial \psi_{t}^{k}}{\partial t} \nabla \phi_{t}^{i}+\psi_{t}^{k} \nabla \frac{\partial \phi_{t}^{i}}{\partial t}\right] d x .
\end{aligned}
$$

Integrating by parts and using equation (4.12), we get

$$
0=\int_{\partial V_{F}(t)} \frac{\partial \psi_{t}^{k}}{\partial t} \cdot n \phi_{t}^{i} d S=-\int_{\partial V_{F}(t)} \nu \frac{\partial \psi_{t}^{k}}{\partial n} \phi_{t}^{i} d S=-\int_{\partial V_{F}(t)}\left(v \nabla \psi_{t}^{k}\right) \cdot n \phi_{t}^{i} d S .
$$

Theorem 4.2. Let $\left\{\psi_{t}^{k}\right\}_{k=1}^{M}$ be the orthonormal eigenfunctions corresponding to a repeated eigenvalue of equation (4.5). Then all values of $\lambda_{t}^{\prime}$ are obtained by solving the eigenvalue problem

$$
\sum_{i=1}^{M} D_{i k} d_{i}=-\lambda_{t}^{\prime} d_{k},
$$


where

$$
D_{i k}:=\eta \int_{\partial V_{F}(t)} \nu \frac{\partial \psi_{t}^{i}}{\partial n} \frac{\partial \psi_{t}^{k}}{\partial n} d S
$$

simultaneously for $\lambda_{t}^{\prime}$ and $d_{k}$.

Remark 4.2. This theorem tells us how repeated eigenvalues evolve. For every eigenvector $d$ of equation (4.18) with corresponding eigenvalue $\lambda^{\prime}$, the function $\sum_{k} d_{k} \psi_{t}^{k}$, which is itself a normalized eigenfunction of equation (4.5) at $t=0$, evolves into an eigenfunction of equation (4.5) at $t=\tau$, where $\tau$ is an infinitesimal quantity, with eigenvalue $\lambda+\tau \lambda^{\prime}$. Without loss of generality, we could choose an orthonormal basis from eigenfunctions of (4.5) such that $D$ is diagonal. With this basis, equation (4.11) holds for all eigenvalues. We will use this fact when proving the error estimate.

Proof. Let

$$
\tilde{\psi}_{t}:=\sum_{k=1}^{M} d_{k} \psi_{t}^{k}
$$

and define $\tilde{\phi}_{t}$ similarly. Note that $\tilde{\psi}_{t}$ obeys equation (4.5) and $\frac{\partial \tilde{\psi}_{t}}{\partial t}$ obeys equation (4.12). Taking the latter, multiplying it by $\psi_{t}^{m}$, and integrating, we get

$$
0=\int_{V_{F}(t)}\left[\eta \Delta \frac{\partial \tilde{\psi}_{t}}{\partial t} \cdot \psi_{t}^{m}-\nabla \frac{\partial \tilde{\phi}_{t}}{\partial t} \cdot \psi_{t}^{m}+\lambda_{t}^{\prime} \tilde{\psi}_{t} \cdot \psi_{t}^{m}+\lambda \frac{\partial \tilde{\psi}_{t}}{\partial t} \cdot \psi_{t}^{m}\right] d x
$$

The term involving $\tilde{\phi}_{t}$ vanishes by equation (4.17). Since $d_{m}=\int_{V_{F}(t)} \tilde{\psi}_{t} \cdot \psi_{t}^{m} d x$, this equals

$$
=\int_{V_{F}(t)}\left[\eta \Delta \frac{\partial \tilde{\psi}_{t}}{\partial t} \cdot \psi_{t}^{m}+\lambda \frac{\partial \tilde{\psi}_{t}}{\partial t} \cdot \psi_{t}^{m}\right] d x-\lambda_{t}^{\prime} d_{m}
$$

Integrating by parts twice, we get

$$
\begin{aligned}
& =\int_{V_{F}(t)}\left[-\eta \nabla \frac{\partial \tilde{\psi}_{t}}{\partial t}: \nabla \psi_{t}^{m}+\lambda \frac{\partial \tilde{\psi}_{t}}{\partial t} \cdot \psi_{t}^{m}\right] d x-\lambda_{t}^{\prime} d_{m} . \\
& =-\eta \int_{\partial V_{F}(t)} \frac{\partial \tilde{\psi}_{t}}{\partial t} \cdot \frac{\partial \psi_{t}^{m}}{\partial n} d S+\int_{V_{F}(t)}\left[\eta \frac{\partial \tilde{\psi}_{t}}{\partial t} \cdot \Delta \psi_{t}^{m}+\lambda \frac{\partial \tilde{\psi}_{t}}{\partial t} \cdot \psi_{t}^{m}\right] d x-\lambda_{t}^{\prime} d_{m},
\end{aligned}
$$

in which the second integral vanishes by using equation (4.12) and noting that

$$
\int_{V_{F}(t)} \frac{\partial \tilde{\psi}_{t}}{\partial t} \cdot \nabla \phi_{t}^{m} d x=0
$$

by Lemma 4.1. Recalling the boundary conditions for $\frac{\partial \tilde{\psi}_{t}}{\partial t}$, we get the desired result.

We will now prove a necessary lemma.

Lemma 4.2. For $i \neq k$,

$$
\left(\frac{\partial \psi_{t}^{k}}{\partial t}, \psi_{t}^{i}\right)_{t}=\frac{-\eta}{\lambda_{t}^{k}-\lambda_{t}^{i}} \int_{\partial V_{F}} \nu \frac{\partial \psi_{t}^{k}}{\partial n} \cdot \frac{\partial \psi_{t}^{i}}{\partial n} d S
$$


Proof. Applying equation (4.8), note that

$$
\begin{aligned}
0 & =\frac{d}{d t} \int_{V_{F}(t)} \nabla \psi_{t}^{k}: \nabla \psi_{t}^{i} d x \\
& =\int_{V_{F}(t)} \frac{\partial}{\partial t}\left(\nabla \psi_{t}^{k}: \nabla \psi_{t}^{i}\right) d x+\int_{\partial V_{F}(t)} \nu \nabla \psi_{t}^{k}: \nabla \psi_{t}^{i} d S
\end{aligned}
$$

Furthermore, integrating by parts and using equation (4.12), note that

$$
\begin{aligned}
\int_{V_{F}(t)} \nabla \frac{\partial \psi_{t}^{k}}{\partial t}: \nabla \psi_{t}^{i} d x & =-\int_{V_{F}(t)} \Delta \frac{\partial \psi_{t}^{k}}{\partial t} \cdot \psi_{t}^{i} d x \\
& =\frac{1}{\eta} \int_{V_{F}(t)}\left[-\nabla \frac{\partial \phi_{t}^{k}}{\partial t}+\lambda_{t}^{\prime, k} \psi_{t}^{k}+\lambda_{t}^{k} \frac{\partial \psi_{t}^{k}}{\partial t}\right] \cdot \psi_{t}^{i} d x .
\end{aligned}
$$

Noting the $L^{2}$-orthogonality of the eigenfunctions as well as equation (4.17), this is

$$
=\frac{\lambda_{t}^{k}}{\eta} \int_{V_{F}} \frac{\partial \psi_{t}^{k}}{\partial t} \cdot \psi_{t}^{i} d x
$$

Next, note that applying equation (4.8) and the boundary conditions $\psi_{t}^{k}=\psi_{t}^{i}=0$ on $\partial V_{F}(t)$, we have

$$
0=\frac{d}{d t} \int_{V_{F}(t)} \psi_{t}^{k} \cdot \psi_{t}^{i} d x=\int_{V_{F}(t)} \frac{\partial}{\partial t}\left(\psi_{t}^{k} \cdot \psi_{t}^{i}\right) d x
$$

Combining equations (4.20)-(4.22), we get the desired result.

\subsection{Error estimate for advected basis}

In the following theorems, we will need to take limits where $\psi_{0}^{k}$ is evaluated outside $V_{F}(0)$. To make such evaluations well defined, we will assume that $\psi_{0}^{k}$ is extended such that $\psi_{0}^{k}$ is $C^{2}$ throughout $V_{F}(t)$ and satisfies $-\eta \Delta \psi_{0}^{k}+\nabla \phi_{0}^{k}=\lambda_{0}^{k} \psi_{0}^{k}$ in $V_{F}(t)$. Note that we do not enforce any boundary conditions on $\psi_{0}^{k}$ on $\partial V_{F}(t)$.

We are now ready to analyze the error in the advected basis.

Theorem 4.3. Let $\left\{\lambda_{t}^{k}\right\}$ be the eigenvalues of equation (4.5) corresponding to the eigenfunctions $\left\{\psi_{t}^{k}\right\}$ at time t. Assume the eigenvalues are ordered such that $0<\lambda_{0}^{1} \leq \lambda_{0}^{2} \leq \ldots$ If $\lambda_{0}^{i}$ is a multiple eigenvalue, assume that the corresponding eigenfunctions satisfy

$$
\int_{\partial V_{F}(0)} \nu \frac{\partial \psi_{0}^{i}}{\partial n} \cdot \frac{\partial \psi_{0}^{j}}{\partial n} d S=0
$$

for all $i, j$ such that $\lambda_{0}^{i}=\lambda_{0}^{j}$. The error in approximating the eigenfunction $\psi_{T}^{k}$ by the advected eigenfunction $\mathcal{A}_{T} \psi_{0}^{k}$ is given by

$$
\begin{aligned}
\lim _{\tau \rightarrow 0} \lim _{h \rightarrow 0} & \frac{\left\|\psi_{T}^{k}-\mathcal{A}_{T} \psi_{0}^{k}\right\|_{\left(L^{2}\left(V_{F}(T)\right)\right)^{d}}}{h^{2}} \\
\leq & \frac{1}{\eta}\left(\frac{\omega_{d}(d-1)}{(2 \pi)^{d}}\right)^{\frac{2}{d}} \int_{0}^{T}\left[4 \beta \lambda_{t}^{k}\|e(v)\|_{\left(L^{\infty}\left(V_{F}(t)\right)\right)^{d \times d}}+2 \sqrt{\lambda_{t}^{k}}\|\Delta v\|_{\left(L^{\infty}\left(V_{F}(t)\right)\right)^{d}}\right. \\
& \left.+\eta\|\nabla \Delta v\|_{\left(L^{\infty}\left(V_{F}(t)\right)\right)^{d \times d}}\right] d t
\end{aligned}
$$

where

$$
\beta:=\sup _{k} \frac{\left\|\Delta \psi_{0}^{k}\right\|_{\left(L^{2}\left(V_{F}(0)\right)\right)^{d}}}{\lambda_{0}^{k}} \leq C_{0}
$$


and $C_{0}$ is the constant in equation (3.1),

$$
\begin{aligned}
\lim _{\tau \rightarrow 0} \lim _{h \rightarrow 0} & \frac{\left\|\nabla\left(\psi_{T}^{k}-\mathcal{A}_{T} \psi_{0}^{k}\right)\right\|_{\left(L^{2}\left(V_{F}(T)\right)\right)^{d \times d}}}{h} \\
\leq & \frac{1}{\sqrt{\eta}}\left(\frac{\omega_{d}(d-1)}{(2 \pi)^{d}}\right)^{\frac{1}{d}} \int_{0}^{T}\left[4 \beta \lambda_{t}^{k}\|e(v)\|_{\left(L^{\infty}\left(V_{F}(t)\right)\right)^{d \times d}}+2 \sqrt{\lambda_{t}^{k}}\|\Delta v\|_{\left(L^{\infty}\left(V_{F}(t)\right)\right)^{d}}\right. \\
& \left.+\eta\|\nabla \Delta v\|_{\left(L^{\infty}\left(V_{F}(t)\right)\right)^{d \times d}}\right] d t .
\end{aligned}
$$

Proof. First, note that doing a Taylor expansion in $\tau$, we can write

$$
\begin{aligned}
A_{\tau} \psi_{0}^{k} & =\left[I+\tau \nabla v_{0}\left(\left(x+v_{0}(x) \tau\right)^{-1}\right)\right] \psi_{0}^{k}\left(\left(x+v_{0}(x) \tau\right)^{-1}\right) \\
& =\left[I+\tau \nabla v_{0}(x)+\mathcal{O}\left(\tau^{2}\right)\right]\left[\psi_{0}^{k}(x)-\tau \nabla \psi_{0}^{k}(x) v_{0}(x)+\mathcal{O}\left(\tau^{2}\right)\right] \\
& =\psi_{0}^{k}(x)+\tau\left[v_{0}, \psi_{0}^{k}\right]+\mathcal{O}\left(\tau^{2}\right) .
\end{aligned}
$$

Also, note that

$$
\psi_{\tau}^{k}=\psi_{0}^{k}+\tau \frac{\partial \psi_{0}^{k}}{\partial t}+\mathcal{O}\left(\tau^{2}\right) .
$$

Therefore, there exists $C>0$ such that

$$
\left\|\psi_{\tau}^{k}-A_{\tau} \psi_{0}^{k}-\tau E^{0, k}\right\|_{\left(L^{2}\left(V_{F}(0)\right)\right)^{d}} \leq C \tau^{2}
$$

and

$$
\left\|\nabla\left(\psi_{\tau}^{k}-A_{\tau} \psi_{0}^{k}-\tau E^{0, k}\right)\right\|_{\left(L^{2}\left(V_{F}(0)\right)\right)^{d \times d}} \leq C \tau^{2},
$$

where

$$
E^{0, k}:=\frac{\partial \psi_{0}^{k}}{\partial t}+\left[v_{0}, \psi_{0}^{k}\right]
$$

Since $\nabla \cdot E^{0, k}=0$ in $V_{F}(0)$ and $E^{0, k}=0$ on $\partial V_{F}(0)$ (from the boundary conditions in equation (4.12)), we can write

$$
E^{0, k}=\sum_{i=1}^{\infty} a_{i}^{k} \psi_{0}^{i}
$$

where

$$
a_{i}^{k}=\left(E^{0, k}, \psi_{0}^{i}\right)
$$

Note that for $i \neq k$, from Lemma 4.2 ,

$$
\left(\frac{\partial \psi_{t}^{k}}{\partial t}, \psi_{t}^{i}\right)_{t}=\frac{-\eta}{\lambda_{t}^{k}-\lambda_{t}^{i}} \int_{\partial V_{F}} \nu \frac{\partial \psi_{t}^{k}}{\partial n} \cdot \frac{\partial \psi_{t}^{i}}{\partial n} d S
$$

When $i=k$, we have

$$
\left(\frac{\partial \psi_{t}^{k}}{\partial t}, \psi_{t}^{k}\right)_{t}=0
$$

from equation (4.16). Now, using equation (4.5) and integrating by parts twice, we have

$$
\left(v \nabla \psi_{t}^{k}, \psi_{t}^{i}\right)_{t}=-\frac{\eta}{\lambda_{t}^{i}} \int_{V_{F}} v \nabla \psi_{t}^{k} \cdot \Delta \psi_{t}^{i} d x+\frac{1}{\lambda_{t}^{i}} \int_{V_{F}} v \nabla \psi_{t}^{k} \cdot \nabla \phi_{t}^{i} d x
$$




$$
\begin{aligned}
= & \frac{\eta}{\lambda_{t}^{i}} \int_{V_{F}}\left[\left(\nabla \psi_{t}^{k} \nabla v\right): \nabla \psi_{t}^{i}+\left(v \nabla^{2} \psi_{t}^{k}\right): \nabla \psi_{t}^{i}\right] d x \\
& -\frac{1}{\lambda_{t}^{i}} \int_{V_{F}} \nabla \psi_{t}^{k}:(\nabla v)^{T} \phi_{t}^{i} d x+\frac{1}{\lambda_{t}^{i}} \int_{\partial V_{F}}\left(v \nabla \psi_{t}^{k}\right) \cdot n \phi_{t}^{i} d S \\
& -\frac{\eta}{\lambda_{t}^{i}} \int_{\partial V_{F}} v \nabla \psi_{t}^{k} \frac{\partial \psi_{t}^{i}}{\partial n} d S .
\end{aligned}
$$

Integrating by parts yet again and using equation (4.5), we get

$$
\begin{aligned}
= & \frac{\eta}{\lambda_{t}^{i}} \int_{V_{F}}\left[\left(\nabla \psi_{t}^{k} \nabla v\right): \nabla \psi_{t}^{i}-\left(\nabla v \nabla^{2} \psi_{t}^{k}\right) \cdot \psi_{t}^{i}+\frac{\lambda_{t}^{k}}{\eta}\left(v \nabla \psi_{t}^{k}\right) \cdot \psi_{t}^{i}\right. \\
& \left.-\frac{1}{\eta}\left(v \nabla^{2} \phi_{t}^{k}\right) \cdot \psi_{t}^{i}\right] d x-\frac{1}{\lambda_{t}^{i}} \int_{V_{F}} \nabla \psi_{t}^{k}:(\nabla v)^{T} \phi_{t}^{i} d x \\
& +\frac{1}{\lambda_{t}^{i}} \int_{\partial V_{F}}\left(v \nabla \psi_{t}^{k}\right) \cdot n \phi_{t}^{i} d S-\frac{\eta}{\lambda_{t}^{i}} \int_{\partial V_{F}} \nu \frac{\partial \psi_{t}^{k}}{\partial n} \cdot \frac{\partial \psi_{t}^{i}}{\partial n} d S \\
= & \frac{\eta}{\lambda_{t}^{i}} \int_{V_{F}}\left[\left(\nabla \psi_{t}^{k} \nabla v\right): \nabla \psi_{t}^{i}+\left(\nabla \psi_{t}^{i} \nabla v\right): \nabla \psi_{t}^{k}+\frac{\lambda_{t}^{k}}{\eta}\left(v \nabla \psi_{t}^{k}\right) \cdot \psi_{t}^{i}\right] d x \\
& +\frac{1}{\lambda_{t}^{i}} \int_{V_{F}} \psi_{t}^{i} \nabla v^{T} \nabla \phi_{t}^{k} d x-\frac{1}{\lambda_{t}^{i}} \int_{V_{F}} \nabla \psi_{t}^{k}:(\nabla v)^{T} \phi_{t}^{i} d x \\
& +\frac{1}{\lambda_{t}^{i}} \int_{\partial V_{F}}\left(v \nabla \psi_{t}^{k}\right) \cdot n \phi_{t}^{i} d S-\frac{\eta}{\lambda_{t}^{i}} \int_{\partial V_{F}} \nu \frac{\partial \psi_{t}^{k}}{\partial n} \cdot \frac{\partial \psi_{t}^{i}}{\partial n} d S .
\end{aligned}
$$

From Lemma 4.1, we know that

$$
\int_{\partial V_{F}}\left(v \nabla \psi_{t}^{k}\right) \cdot n \phi_{t}^{i} d S=0 .
$$

Therefore,

$$
\begin{aligned}
\left(v \nabla \psi_{t}^{k}, \psi_{t}^{i}\right)_{t}= & \frac{1}{\lambda_{t}^{i}-\lambda_{t}^{k}}\left[-\eta \int_{\partial V_{F}} \nu \frac{\partial \psi_{t}^{k}}{\partial n} \cdot \frac{\partial \psi_{t}^{i}}{\partial n} d S+\eta \int_{V_{F}}\left[\nabla \psi_{t}^{k}\left(\nabla v+(\nabla v)^{T}\right)\right]: \nabla \psi_{t}^{i} d x\right. \\
& \left.+\int_{V_{F}} \psi_{t}^{i} \nabla v^{T} \nabla \phi_{t}^{k} d x-\int_{V_{F}} \nabla \psi_{t}^{k}:(\nabla v)^{T} \phi_{t}^{i} d x\right] .
\end{aligned}
$$

In a similar fashion, using equation (4.5) and integrating by parts several times, we have

$$
\begin{aligned}
\left(\psi_{t}^{k} \nabla v, \psi_{t}^{i}\right)_{t}= & -\frac{\eta}{\lambda_{t}^{i}} \int_{V_{F}}\left(\Delta \psi_{t}^{i} \nabla v\right) \cdot \psi_{t}^{k} d x+\frac{1}{\lambda_{t}^{i}} \int_{V_{F}} \psi_{t}^{k} \nabla v^{T} \nabla \phi_{t}^{i} d x \\
= & \frac{\eta}{\lambda_{t}^{i}} \int_{V_{F}}\left[\left(\nabla v \nabla \psi_{t}^{i}\right): \nabla \psi_{t}^{k}+\left(\nabla^{2} v \psi_{t}^{k}\right): \nabla \psi_{t}^{i}\right] d x-\frac{1}{\lambda_{t}^{i}} \int_{V_{F}} \nabla \psi_{t}^{k}: \nabla v^{T} \phi_{t}^{i} d x \\
= & -\frac{\eta}{\lambda_{t}^{i}} \int_{V_{F}}\left[\left(\Delta \psi_{t}^{k} \nabla v\right) \cdot \psi_{t}^{i}+\left(\nabla^{2} v \psi_{t}^{i}\right): \nabla \psi_{t}^{k}-\left(\nabla^{2} v \psi_{t}^{k}\right): \nabla \psi_{t}^{i}\right] d x \\
& -\frac{1}{\lambda_{t}^{i}} \int_{V_{F}} \nabla \psi_{t}^{k}: \nabla v^{T} \phi_{t}^{i} d x \\
= & \frac{\lambda_{t}^{k}}{\lambda_{t}^{i}}\left(\psi_{t}^{k} \nabla v, \psi_{t}^{i}\right)_{t}-\frac{1}{\lambda_{t}^{i}} \int_{V_{F}} \psi_{t}^{i} \nabla v^{T} \nabla \phi_{t}^{k} d x-\frac{1}{\lambda_{t}^{i}} \int_{V_{F}} \nabla \psi_{t}^{k}: \nabla v^{T} \phi_{t}^{i} d x \\
& +\frac{\eta}{\lambda_{t}^{i}} \int_{V_{F}}\left[\left(\nabla^{2} v \nabla \psi_{t}^{i}\right) \cdot \psi_{t}^{k}-\left(\nabla \psi_{t}^{k} \nabla^{2} v\right) \cdot \psi_{t}^{i}\right] d x
\end{aligned}
$$


Combining equations (4.30)-(4.33), we get that, for $i \neq k$,

$$
\begin{aligned}
a_{i}^{k}= & \frac{1}{\lambda_{0}^{i}-\lambda_{0}^{k}}\left[-2 \eta \int_{V_{F}(0)} \nabla \psi_{0}^{k}\left(\nabla \psi_{0}^{i}\right)^{T}: e(v) d x+2 \int_{V_{F}(0)} \psi_{0}^{i} \nabla \phi_{0}^{k}: e(v) d x\right. \\
& \left.-\eta \int_{V_{F}(0)}\left[\left(\nabla^{2} v \nabla \psi_{0}^{i}\right) \cdot \psi_{0}^{k}+\left(\nabla \psi_{0}^{k} \nabla^{2} v\right) \cdot \psi_{0}^{i}\right] d x\right]
\end{aligned}
$$

and, for $i=k$,

$$
a_{k}^{k}=-\int_{V_{F}(0)}\left(\psi_{0}^{k} e(v)\right) \cdot \psi_{0}^{k} d x .
$$

Using equation (4.5) and integrating by parts, we rewrite equation (4.34) slightly:

$$
\begin{aligned}
a_{i}^{k}= & \frac{1}{\lambda_{0}^{i}-\lambda_{0}^{k}}\left[2 \eta \int_{V_{F}(0)}\left(\psi_{0}^{i} \nabla^{2} \psi_{0}^{k}\right): e(v) d x+2 \eta \int_{V_{F}(0)}\left(\nabla \psi_{0}^{k} \Delta v\right) \cdot \psi_{0}^{i} d x+2 \int_{V_{F}(0)} \psi_{0}^{i} \nabla \phi_{0}^{k}: e(v) d x\right. \\
& \left.+\eta \int_{V_{F}(0)} \psi_{0}^{k} \psi_{0}^{i}: \nabla \Delta v d x\right] .
\end{aligned}
$$

In light of equations (4.4), (4.1), and (4.25), $\mathcal{A}_{\tau}=1+\mathcal{O}(\tau)$. Therefore, $\exists C>0$ such that

$$
\begin{aligned}
\left\|\psi_{t_{n}}^{k}-\mathcal{A}_{t_{n}} \psi_{0}^{k}\right\|_{\left(H^{1}\left(V_{F}\left(t_{n}\right)\right)\right)^{d}} & \leq \sum_{i=1}^{n}\left\|\mathcal{A}_{(n-i) \tau}\left(\psi_{t_{i}}^{k}-\mathcal{A}_{\tau} \psi_{t_{i-1}}^{k}\right)\right\|_{\left(H^{1}\left(V_{F}\left(t_{n}\right)\right)\right)^{d}} \\
& \leq \sum_{i=1}^{n}\left\|\psi_{t_{i}}^{k}-\mathcal{A}_{\tau} \psi_{t_{i-1}}^{k}\right\|_{\left(H^{1}\left(V_{F}\left(t_{i}\right)\right)\right)^{d}}+C \tau^{2} .
\end{aligned}
$$

Therefore, noting equations (4.4), (4.27), (4.29), (4.36), and (4.35), we have that $\exists C>0$ such that

$$
\begin{aligned}
& \left\|\psi_{t_{n}}^{k}-\mathcal{A}_{t_{n}} \psi_{0}^{k}\right\|_{\left(L^{2}\left(V_{F}(0)\right)\right)^{d}} \leq \tau \sum_{i=1}^{N} \sqrt{\sum_{j=M+1}^{\infty}\left(a_{j}^{k}\left(t_{i}\right)\right)^{2}}+C \tau^{2} \\
& \quad \leq \tau \sum_{i=1}^{N} \frac{1}{\lambda_{t_{i}}^{M+1}-\lambda_{t_{i}}^{k}}\left[4 \beta \lambda_{t_{i}}^{k}\left\|e\left(v_{t_{i}}\right)\right\|_{L^{\infty}}+2 \sqrt{\lambda_{t_{i}}^{k}}\left\|\Delta v_{t_{i}}\right\|_{L^{\infty}}+\eta\left\|\nabla \Delta v_{t_{i}}\right\|_{L^{\infty}}\right]+C \tau^{2} .
\end{aligned}
$$

where

$$
\beta:=\sup _{k} \frac{\left\|\Delta \psi_{0}^{k}\right\|_{\left(L^{2}\left(V_{F}(0)\right)\right)^{d}}}{\lambda_{0}^{k}} \leq C_{0}
$$

and $C_{0}$ is the constant in equation (3.1). Similarly,

$$
\begin{aligned}
& \left\|\nabla \psi_{t_{n}}^{k}-\nabla \mathcal{A}_{t_{n}} \psi_{0}^{k}\right\|_{\left(L^{2}\left(V_{F}(0)\right)\right)^{d}} \leq \tau \sum_{i=1}^{N} \sqrt{\sum_{j=M+1}^{\infty} \lambda_{t_{i}}^{j}\left(a_{j}^{k}\left(t_{i}\right)\right)^{2}}+C \tau^{2} \\
& \quad \leq \tau \sum_{i=1}^{N} \frac{\sqrt{\lambda_{t_{i}}^{M+1}}}{\lambda_{t_{i}}^{M+1}-\lambda_{t_{i}}^{k}}\left[4 \beta \lambda_{t_{i}}^{k}\left\|e\left(v_{t_{i}}\right)\right\|_{L^{\infty}}+2 \sqrt{\lambda_{t_{i}}^{k}}\left\|\Delta v_{t_{i}}\right\|_{L^{\infty}}+\eta\left\|\nabla \Delta v_{t_{i}}\right\|_{L^{\infty}}\right]+C \tau^{2} .
\end{aligned}
$$

Noting equation (3.5) and taking the limits $h \rightarrow 0$ and $\tau \rightarrow 0$ in equations (4.37) and (4.38), we get the desired result. 


\subsection{Full error estimate}

Theorem 4.4. Let $u$ solve equation (2.2), and let $u_{h}(T)=\sum_{k=1}^{M} a_{k} \psi_{T}^{k}$ be the Galerkin solution of equation (2.2) in $V^{h}\left(V_{F}(T)\right)$. Let $\tilde{u}_{h}:=\sum_{k=1}^{M} a_{k} \mathcal{A}_{T} \psi_{0}^{k}$. Then

$$
\begin{aligned}
\| u(T) & -\hat{u}_{h}(T) \|_{\left(H^{1}\left(V_{F}(T)\right)\right)^{d}} \\
& \leq\left\|u(T)-\tilde{u}_{h}(T)\right\|_{\left(H^{1}\left(V_{F}(T)\right)\right)^{d}} \\
& \leq\left\|u(T)-u_{h}(T)\right\|_{\left(H^{1}\left(V_{F}(T)\right)\right)^{d}}+\left\|u_{h}(T)-\tilde{u}_{h}(T)\right\|_{\left(H^{1}\left(V_{F}(T)\right)\right)^{d}},
\end{aligned}
$$

where

$$
\lim _{h \rightarrow 0} \frac{\left\|u(T)-u_{h}(T)\right\|_{\left(H^{1}\left(V_{F}(T)\right)\right)^{d}}}{h\|f\|_{\left(L^{2}\left(V_{F}(T)\right)\right)^{d}}}=\frac{1}{\sqrt{\eta}}\left(\frac{\omega_{d}(d-1)}{(2 \pi)^{d}}\right)^{\frac{1}{d}}
$$

and

$$
\begin{aligned}
& \lim _{\tau \rightarrow 0} \lim _{h \rightarrow 0} \frac{\left\|u_{h}(T)-\tilde{u}_{h}(T)\right\|_{\left(H^{1}\left(V_{F}(T)\right)\right)^{d}}}{h\|f\|_{\left(H^{(2 d-1) / 2}\left(V_{F}(T)\right)\right)^{d}}} \\
& \leq \frac{2 \beta d\left|V_{F}\right|^{1 / d}}{(2 \pi)^{1 / 2+d} \eta^{1 / 4+d}\left((d-1)\left|V_{F}\right| \omega_{d}\right)^{1+1 / 2 d}} \int_{0}^{T}\|e(v(t))\|_{\left(L^{\infty}\left(V_{F}(t)\right)\right)^{d \times d}} d t .
\end{aligned}
$$

Proof. Equation (4.39) follows from the triangle inequality and the definition of $\hat{u}_{h}$. Equation (4.40) follows from equation (3.4). Note that

$$
\begin{aligned}
\left\|u_{h}\left(t_{n}\right)-\tilde{u}_{h}\left(t_{n}\right)\right\|_{\left(H^{1}\left(V_{F}\left(t_{n}\right)\right)\right)^{d}} & =\left\|\sum_{k=1}^{M} a_{k}\left(\psi_{t_{n}}^{k}-\mathcal{A}_{t_{n}} \psi_{0}^{k}\right)\right\|_{\left(H^{1}\left(V_{F}\left(t_{n}\right)\right)\right)^{d}} \\
& \left.=\left\|\sum_{k=1}^{M}\left(\lambda_{t_{n}}^{k}\right)^{1+\alpha / 2} a_{k} \frac{\psi_{t_{n}}^{k}-\mathcal{A}_{t_{n}} \psi_{0}^{k}}{\left(\lambda_{t_{n}}^{k}\right)^{1+\alpha / 2}}\right\|_{\left(H^{1}\left(V_{F}\left(t_{n}\right)\right)\right)^{d}}\right)^{\frac{1}{2}} \\
& \leq\left(\sum_{k=1}^{M}\left(\lambda_{t_{n}}^{k}\right)^{2+\alpha} a_{k}^{2}\right)^{\frac{1}{2}}\left(\sum_{k=1}^{M}\left\|\frac{\psi_{t_{n}}^{k}-\mathcal{A}_{t_{n}} \psi_{0}^{k}}{\left(\lambda_{t_{n}}^{k}\right)^{1+\alpha / 2}}\right\|_{\left(H^{1}\left(V_{F}\left(t_{n}\right)\right)\right)^{d}}^{2}\right. \\
& \leq\|f\|_{\left(H^{\alpha}\left(V_{F}\left(t_{n}\right)\right)\right)^{d}}\left(\sum_{k=1}^{M} \frac{\left\|\psi_{t_{n}}^{k}-\mathcal{A}_{t_{n}} \psi_{0}^{k}\right\|_{\left(H^{1}\left(V_{F}\left(t_{n}\right)\right)\right)^{d}}^{2}}{\left(\lambda_{t_{n}}^{k}\right)^{2+\alpha}}\right)^{\frac{1}{2}} .
\end{aligned}
$$

Using equation (4.38), we have

$$
\begin{aligned}
& \sum_{k=1}^{M} \frac{\left\|\psi_{t_{n}}^{k}-\mathcal{A}_{t_{n}} \psi_{0}^{k}\right\|_{\left(H^{1}\left(V_{F}\left(t_{n}\right)\right)\right)^{d}}^{2}}{\left(\lambda_{t_{n}}^{k}\right)^{2+\alpha}} \\
& \quad \leq \tau^{2} \sum_{i=1}^{N} \sum_{k=1}^{M} \frac{\lambda_{t_{i}}^{M+1}}{\left(\lambda_{t_{i}}^{M+1}-\lambda_{t_{i}}^{k}\right)^{2}\left(\lambda_{t_{n}}^{k}\right)^{2+\alpha}}\left[16 \beta^{2}\left(\lambda_{t_{i}}^{k}\right)^{2}\left\|e\left(v_{t_{i}}\right)\right\|_{L^{\infty}}^{2}+4 \lambda_{t_{i}}^{k}\left\|\Delta v_{t_{i}}\right\|_{L^{\infty}}^{2}+\eta^{2} \lambda_{t_{i}}^{k}\left\|\nabla \Delta v_{t_{i}}\right\|_{L^{\infty}}^{2}\right]+C \tau^{4} \\
& \quad \leq \tau^{2} \sum_{i=1}^{N} \int_{1}^{M} \frac{\lambda_{t_{i}}^{M+1}}{\left(\lambda_{t_{i}}^{M+1}-\lambda_{t_{i}}^{k}\right)^{2}\left(\lambda_{t_{n}}^{k}\right)^{2+\alpha}}\left[16 \beta^{2}\left(\lambda_{t_{i}}^{k}\right)^{2}\left\|e\left(v_{t_{i}}\right)\right\|_{L^{\infty}}^{2}+4 \lambda_{t_{i}}^{k}\left\|\Delta v_{t_{i}}\right\|_{L^{\infty}}^{2}+\eta^{2} \lambda_{t_{i}}^{k}\left\|\nabla \Delta v_{t_{i}}\right\|_{L^{\infty}}^{2}\right] d k+C \tau^{4}
\end{aligned}
$$

Setting $\alpha=\frac{2 d-1}{2}$, applying Theorem 4.3, equation (3.5), and taking the limit $\tau \rightarrow 0$, we get equation (4.41). 


\section{Basis localization}

Solutions to equation (3.2) are expensive to compute, so we will simplify the problem by using solutions to localized problems.

\subsection{Stokes Neumann eigenfunctions}

For $D(t) \subset V_{F}(t)$, let $S_{D}(t)$ denote the closure of the set

$$
\left\{v \in\left(C^{\infty}(D(t))\right)^{d} \mid \nabla \cdot v=0, v \cdot n=0 \text { for } x \in \partial D(t) \cap \partial V_{F}(t)\right\}
$$

in the $\left(L^{2}\left(V_{F}(t)\right)\right)^{d}$ norm and define $S_{D}^{m}(t):=S_{D}(t) \cap\left(H^{m}(D(t))\right)^{d}$ for $m \in \mathbb{N}$.

The following theorem is established in [9].

Theorem 5.1. Let $t \in(0, \infty)$ be fixed. For all $f \in S_{D}^{m}(t)$ and $m \in \mathbb{N}$, the equation

$$
\left\{\begin{array}{lr}
-\eta \Delta u+\nabla p=f, \nabla \cdot u=0 & x \in D(t) \\
u=0 & x \in \partial D(t) \cap \partial V_{F}(t) \\
\sigma(u) n=0 & x \in \partial D(t) \backslash \partial V_{F}(t)
\end{array}\right.
$$

has a unique solution $u \in S_{D}^{m+2}(t)$ with $p \in H^{m+1}\left(V_{F}(t)\right)$. Furthermore, $\exists C_{m}>0$ such that $(u, p)$ satisfies

$$
\|u\|_{\left(H^{m+2}\left(V_{F}(t)\right)\right)^{d}}+\|p\|_{H^{m+1}\left(V_{F}(t)\right)} \leq C_{m}\|f\|_{\left.H^{m}\left(V_{F}(t)\right)\right)^{d}} .
$$

Once again, the solution operator $\mathcal{S}_{D}^{-1}(t): S_{D}(t) \rightarrow S_{D}(t) \supset S_{D}^{2}(t)$ to equation (5.1) is compact. Therefore, the eigenfunctions $\left(\psi_{D, t}^{k}, \phi_{D, t}^{k}\right)$ satisfying

$$
\left\{\begin{array}{lr}
-\eta \Delta \psi_{D, t}^{k}+\nabla \phi_{D, t}^{k}=\lambda_{D, t}^{k} \psi_{D, t}^{k}, \nabla \cdot \psi_{D, t}^{k}=0 & x \in D \\
\psi_{D, t}^{k}=0 & x \in \partial D \cap \partial V_{F} \\
\sigma\left(\psi_{D, t}^{k}\right) n=0 & x \in \partial D \backslash \partial V_{F}
\end{array}\right.
$$

form a complete basis for $S_{D}(t)$. Furthermore, the set of eigenvalues $\left\{\lambda_{D, t}^{k}\right\}$ is countable, and $\lambda_{D, t}^{k} \geq 0$ for all $k$.

We will need the following

Lemma 5.1. Let $u \in\left(H_{0}^{1}\left(V_{F}\right)\right)^{d} \cap\left(H^{2}\left(V_{F}\right)\right)^{d}$. Then

$$
\lim _{M \rightarrow \infty}\left\|u-\sum_{k=1}^{M} c_{k} \psi_{D, t}^{k}\right\|_{\left(H^{1}(D)\right)^{d}}=0
$$

where $c_{k}:=\left(u, \psi_{D, t}^{k}\right)_{\left(L^{2}(D)\right)^{d}}$.

Proof. That

$$
\lim _{M \rightarrow \infty}\left\|u-\sum_{k=1}^{M} c_{k} \psi_{D, t}^{k}\right\|_{\left(L^{2}(D)\right)^{d}}=0
$$

is a standard result [3]. Let $u^{\epsilon} \in\left(C^{\infty}(\bar{D})\right)^{d}$ be such that $\nabla \cdot u^{\epsilon}=0, u^{\epsilon}(x)=0$ for all $x \in \partial D \cap \partial V_{F}, \frac{\partial u^{\epsilon}}{\partial n}=0$ for all $x \in \partial D \backslash \partial V_{F}$, and $\left\|u^{\epsilon}-u\right\|_{\left(H^{1}(D)\right)^{d}}<\epsilon$; let $c_{k}^{\epsilon}:=\left(u_{m}, \psi_{D, t}^{k}\right)_{\left(L^{2}(D)\right)^{d}}$, and let $u_{m}^{\epsilon}:=\sum_{k=1}^{m} c_{k}^{\epsilon} \psi_{D, t}^{k}$. By the variational form of the eigenvalue problem (5.3) and the triangle inequality, we have

$$
\begin{gathered}
\left\|\nabla \sum_{k=1}^{m} c_{k} \psi_{D, t}^{k}-\nabla u\right\|_{\left(L^{2}(D)\right)^{d \times d}} \leq\left\|\nabla \sum_{k=1}^{m} c_{k}^{\epsilon} \psi_{D, t}^{k}-\nabla u\right\|_{\left(L^{2}(D)\right)^{d \times d}} \\
\leq\left\|\nabla u-\nabla u^{\epsilon}\right\|_{\left(L^{2}(D)\right)^{d \times d}}+\left\|\nabla u^{\epsilon}-\nabla \sum_{k=1}^{m} c_{k}^{\epsilon} \psi_{D, t}^{k}\right\|_{\left(L^{2}(D)\right)^{d \times d}} .
\end{gathered}
$$


The first term is bounded by $\epsilon$. To control the second term, note that

$$
\left\|\nabla u^{\epsilon}\right\|_{\left(L^{2}(D)\right)^{d \times d}}=\left\|\nabla u^{\epsilon}-\nabla u_{m}^{\epsilon}+\nabla u_{m}^{\epsilon}\right\|_{\left(L^{2}(D)\right)^{d \times d}} .
$$

Furthermore, since $\left(\nabla u^{\epsilon}-\nabla u_{m}^{\epsilon}, \nabla u_{m}^{\epsilon}\right)_{\left(L^{2}(D)\right)^{d \times d}}=0$, we have

$$
\left\|\nabla u^{\epsilon}\right\|_{\left(L^{2}(D)\right)^{d \times d}}=\left\|\nabla u^{\epsilon}-\nabla u_{m}^{\epsilon}\right\|_{\left(L^{2}(D)\right)^{d \times d}}+\left\|\nabla u_{m}^{\epsilon}\right\|_{\left(L^{2}(D)\right)^{d \times d}} .
$$

Therefore, we need only show that $\left\|\nabla u_{m}^{\epsilon}\right\|_{\left(L^{2}(D)\right)^{d \times d}} \rightarrow\left\|\nabla u^{\epsilon}\right\|_{\left(L^{2}(D)\right) d \times d}$ as $m \rightarrow \infty$. Since

$$
\left\|\nabla u_{m}^{\epsilon}\right\|_{\left(L^{2}(D)\right)^{d \times d}}^{2}=\sum_{k=1}^{M} \lambda_{D, t}^{k}\left(c_{k}^{\epsilon}\right)^{2},
$$

this is equivalent to showing

$$
\left\|\nabla u^{\epsilon}\right\|_{\left(L^{2}(D)\right)^{d \times d}}^{2}=\sum_{k=1}^{\infty} \lambda_{D, t}^{k}\left(c_{k}^{\epsilon}\right)^{2} .
$$

Let $g^{\epsilon}:=-\eta \Delta u^{\epsilon}$ for $x \in D$. Then, since either $u^{\epsilon}$ or $\frac{\partial u^{\epsilon}}{\partial n}=0$ everywhere on $\partial D$, we have

$$
\begin{aligned}
\left\|\nabla u^{\epsilon}\right\|_{\left(L^{2}(D)\right)^{d \times d}}^{2} & =-\left(\Delta u^{\epsilon}, u^{\epsilon}\right)_{\left(L^{2}(D)\right)^{d}}+\int_{\partial D} u^{\epsilon} \cdot \frac{\partial u^{\epsilon}}{\partial n} d S \\
& =\frac{1}{\eta}\left(g^{\epsilon}, u^{\epsilon}\right)_{\left(L^{2}(D)\right)^{d}} .
\end{aligned}
$$

Furthermore, integrating by parts several times and using the boundary conditions on $u$ and $\psi_{D, t}^{k}$, we have

$$
\begin{aligned}
\left(g^{\epsilon}, \psi_{D, t}^{k}\right)_{\left(L^{2}(D)\right)^{d}}= & -\eta\left(\Delta u^{\epsilon}, \psi_{D, t}^{k}\right)_{\left(L^{2}(D)\right)^{d}} \\
= & \eta\left(\nabla u^{\epsilon}, \nabla \psi_{D, t}^{k}\right)_{\left(L^{2}(D)\right)^{d \times d}}-\eta \int_{\partial D} \frac{\partial u^{\epsilon}}{\partial n} \cdot \psi_{D, t}^{k} d S \\
= & \left(u^{\epsilon},-\eta \Delta \psi_{D, t}^{k}+\nabla \phi_{D, t}^{k}\right)_{\left(L^{2}(D)\right)^{d}}+\left(\nabla \cdot u^{\epsilon}, \phi_{D, t}^{k}\right)_{\left(L^{2}(D)\right)} \\
& +\int_{\partial D}\left[\sigma\left(\psi_{D, t}^{k}\right) n \cdot u^{\epsilon}-\frac{\partial u^{\epsilon}}{\partial n} \cdot \psi_{D, t}^{k}\right] d S \\
= & \lambda_{D, t}^{k} c_{k}^{\epsilon} .
\end{aligned}
$$

Noting that [3]

$$
\left(g^{\epsilon}, u^{\epsilon}\right)_{\left(L^{2}(D)\right)^{d}}=\sum_{k=1}^{\infty}\left(g^{\epsilon}, \psi_{D, t}^{k}\right)_{\left(L^{2}(D)\right)^{d}}\left(u^{\epsilon}, \psi_{D, t}^{k}\right)_{\left(L^{2}(D)\right)^{d}},
$$

we then get that

$$
\left\|\nabla u^{\epsilon}\right\|_{\left(L^{2}(D)\right) d \times d}^{2}=\frac{1}{\eta} \sum_{k=1}^{\infty} \lambda_{D, t}^{k}\left(c_{k}^{\epsilon}\right)^{2},
$$

as desired.

\subsection{Localized basis}

Let $\left\{D_{i}\right\}_{i=1}^{N_{D}}$ be a nonoverlapping decomposition of $V_{F}$. Fix $\delta>0$, and let $D_{i}^{\prime}:=\left\{x \in V_{F} \mid \operatorname{dist}\left(x, D_{i}\right)<\delta\right\}$. Let $\xi_{i}$ be a partition of unity subordinate to $\left\{D_{i}^{\prime}\right\}$. From Lemma 5.1, we can define a localized basis by using 
the functions $\left\{\xi_{i} \psi_{D_{i}^{\prime}}^{k}\right\}$ for $i=1, \ldots, N_{D}$ and $k=1, \ldots, h^{-d}\left|D_{i}^{\prime}\right|$. Let $A_{t_{i}}$ be defined as in equation (4.1). We can advect the eigenfunctions as before, by defining the evolution operator $\mathcal{A}_{t_{i}}$ via

$$
\mathcal{A}_{t_{i+1}} \psi_{D, 0}^{k}(x):=A_{\tau} \mathcal{A}_{t_{i}} \psi_{D, 0}^{k}(x)+\tau \sum_{j=1}^{M} a_{j}^{k} \mathcal{A}_{t_{i+1}} \psi_{D, 0}^{j},
$$

where, for $j \neq k$,

$$
\begin{aligned}
a_{j}^{k}= & \frac{\eta}{\lambda_{t_{i}}^{j}-\lambda_{t_{i}}^{k}}\left[2 \int_{V_{F}\left(t_{i}\right)}\left(\nabla \mathcal{A}_{t_{i}} \psi_{D, 0}^{j} e\left(v_{t_{i}}\right)\right): \nabla \mathcal{A}_{t_{i}} \psi_{D, 0}^{k} d x\right. \\
& \left.+\int_{V_{F}\left(t_{i}\right)}\left[\left(\nabla^{2} v_{t_{i}} \nabla \mathcal{A}_{t_{i}} \psi_{D, 0}^{j}\right) \cdot \mathcal{A}_{t_{i}} \psi_{D, 0}^{k}-\left(\nabla \mathcal{A}_{t_{i}} \psi_{D, 0}^{k} \nabla^{2} v_{t_{i}}\right) \cdot \mathcal{A}_{t_{i}} \psi_{D, 0}^{j}\right] d x\right]
\end{aligned}
$$

and, for $j=k$,

$$
a_{k}^{k}=-\int_{V_{F}\left(t_{i}\right)}\left(\mathcal{A}_{t_{i}} \psi_{D, 0}^{k} e\left(v_{t_{i}}\right)\right) \cdot \mathcal{A}_{t_{i}} \psi_{D, 0}^{k} d x
$$

The error due to advection is quantified in the following theorem.

Theorem 5.2. Let $\left\{\lambda_{D, t}^{k}\right\}$ be the eigenvalues of equation (5.3) corresponding to the eigenfunctions $\left\{\psi_{D, t}^{k}\right\}$ at time $t$. Assume the eigenvalues are simple and ordered such that $0<\lambda_{D, 0}^{1} \leq \lambda_{D, 0}^{2} \leq \ldots$. The error in approximating the eigenfunction $\psi_{D, T}^{k}$ by the advected eigenfunction $\mathcal{A}_{T} \psi_{D, 0}^{k}$ is given by

$$
\begin{aligned}
& \lim _{\tau \rightarrow 0} \lim _{h \rightarrow 0} \frac{\left\|\psi_{D, T}^{k}-\mathcal{A}_{T} \psi_{D, 0}^{k}\right\|_{\left(L^{2}(D(T))\right)^{d}}}{h^{2}} \\
& \quad \leq\left(\frac{\omega_{d}(d-1)}{(2 \pi)^{d}}\right)^{\frac{2}{d}} \int_{0}^{T}\left[2 \beta \lambda_{D, t}^{k}\|e(v)\|_{\left(L^{\infty}(D(t))\right)^{d \times d}}+4 \sqrt{\lambda_{D, t}^{k}}\left\|\nabla^{2} v\right\|_{\left(L^{\infty}(D(t))\right)^{d \times d \times d}}\right] d t,
\end{aligned}
$$

where

$$
\beta:=\sup _{k} \frac{\left\|\Delta \psi_{0}^{k}\right\|_{\left(L^{2}\left(V_{F}(0)\right)\right)^{d}}}{\lambda_{0}^{k}} \leq C_{0}
$$

and $C_{0}$ is the constant in equation (5.2), and

$$
\begin{aligned}
& \lim _{\tau \rightarrow 0} \lim _{h \rightarrow 0} \frac{\left\|\nabla\left(\psi_{D, T}^{k}-\mathcal{A}_{T} \psi_{D, 0}^{k}\right)\right\|_{\left(L^{2}(D(T))\right)^{d \times d}}}{h} \\
& \quad \leq\left(\frac{\omega_{d}(d-1)}{(2 \pi)^{d}}\right)^{\frac{1}{d}} \int_{0}^{T}\left[2 \beta \lambda_{D, t}^{k}\|e(v)\|_{\left(L^{\infty}(D(t))\right)^{d \times d}}+4 \sqrt{\lambda_{D, t}^{k}}\left\|\nabla^{2} v\right\|_{\left(L^{\infty}(D(t))\right)^{d \times d \times d}}\right] d t .
\end{aligned}
$$

Proof. First, note that doing a Taylor expansion in $\tau$, we can write

$$
\begin{aligned}
A_{\tau} \psi_{0}^{D, k} & =\left[I+\tau \nabla v_{0}\left(\left(x+v_{0}(x) \tau\right)^{-1}\right)\right] \psi_{D, 0}^{k}\left(\left(x+v_{0}(x) \tau\right)^{-1}\right) \\
& =\left[I+\tau \nabla v_{0}(x)+\mathcal{O}\left(\tau^{2}\right)\right]\left[\psi_{D, 0}^{k}(x)-\tau \nabla \psi_{D, 0}^{k}(x) v(x)+\mathcal{O}\left(\tau^{2}\right)\right] \\
& =\psi_{D, 0}^{k}(x)+\tau\left[v_{0}, \psi_{D, 0}^{k}\right]+\mathcal{O}\left(\tau^{2}\right) .
\end{aligned}
$$

Also, note that

$$
\psi_{D, \tau}^{k}=\psi_{D, 0}^{k}+\tau \frac{\partial \psi_{D, 0}^{k}}{\partial t}+\mathcal{O}\left(\tau^{2}\right) .
$$


Therefore, there exists $C>0$ such that

$$
\left\|\psi_{D, \tau}^{k}-A_{\tau} \psi_{D, 0}^{k}-\tau E^{D, 0, k}\right\|_{\left(L^{2}(D(0))\right)^{d}} \leq C \tau^{2}
$$

and

$$
\left\|\nabla\left(\psi_{D, \tau}^{k}-A_{\tau} \psi_{D, 0}^{k}-\tau E^{D, 0, k}\right)\right\|_{\left(L^{2}(D(0))\right) d \times d} \leq C \tau^{2},
$$

where

$$
E^{D, 0, k}:=\frac{\partial \psi_{D, 0}^{k}}{\partial t}+\left[v_{0}, \psi_{D, 0}^{k}\right]
$$

From Lemma 5.1 and the fact that $\nabla \cdot E^{D, 0, k}=0$ in $D(0)$, we can write

$$
E^{D, 0, k}=\sum_{i=1}^{\infty} a_{i}^{k} \psi_{D, 0}^{i}
$$

where

$$
a_{i}^{k}=\left(E^{D, 0, k}, \psi_{D, 0}^{i}\right) .
$$

Using the boundary conditions in (5.3), we get that for $x \in \partial D \backslash \partial V_{F}$,

$$
0=\frac{\delta \sigma n}{\delta t}=\frac{\delta \sigma}{\delta t} n+\sigma \frac{\delta n}{\delta t}
$$

It is shown in [37] that

$$
\frac{\delta n}{\delta t}=-\nabla_{\partial D} \nu
$$

where $\nabla_{\partial D}$ is the gradient operator on $\partial D$. Therefore, for $x \in \partial D \backslash \partial V_{F}$, we have

$$
\frac{\partial \sigma\left(\psi_{D, t}^{k}\right)}{\partial t} n=-\nu n \nabla \sigma\left(\psi_{D, t}^{k}\right) n+\sigma\left(\psi_{D, t}^{k}\right) \nabla_{\partial D} \nu
$$

Using this observation and differentiating equation (5.3), we get

$$
\left\{\begin{array}{lr}
-\eta \Delta \frac{\partial \psi_{D, t}^{k}}{\partial t}+\nabla \frac{\partial \phi_{D, t}^{k}}{\partial t}=\lambda_{D, t}^{\prime, k} \psi_{D, t}^{k}+\lambda_{D, t}^{k} \frac{\partial \psi_{D, t}^{k}}{\partial t}, \nabla \cdot \frac{\partial \psi_{D, t}^{k}}{\partial t}=0 & x \in D \\
\frac{\partial \psi_{D, t}^{k}}{\partial t}=-\nu \frac{\partial \psi_{D, t}^{k}}{\partial n} & x \in \partial D^{D}:=\partial D \cap \partial V_{F} \\
\frac{\partial \sigma\left(\psi_{D, t}^{k}\right)}{\partial t} n=-\nu n \nabla \sigma\left(\psi_{D, t}^{k}\right) n+\sigma\left(\psi_{D, t}^{k}\right) \nabla_{\partial D} \nu & x \in \partial D^{N}:=\partial D \backslash \partial V_{F} .
\end{array}\right.
$$

Therefore, for $i \neq k$, we have

$$
\left(\frac{\partial \psi_{t}^{k}}{\partial t}, \psi_{t}^{i}\right)_{D, t}=\frac{1}{\lambda_{D, t}^{k}} \int_{D}\left[-\nabla \cdot \sigma\left(\frac{\partial \psi_{D, t}^{k}}{\partial t}\right) \cdot \psi_{D, t}^{i}-\lambda_{D, t}^{\prime, k} \psi_{D, t}^{k} \cdot \psi_{D, t}^{i}\right] d x
$$

in which the second term vanishes. Integrating by parts twice and noting that $\psi_{D, t}^{i}=0$ on $\partial D^{D}$, we get

$$
\begin{aligned}
& =\frac{1}{\lambda_{D, t}^{k}}\left[\int_{D} \eta\left(\nabla \frac{\partial \psi_{D, t}^{k}}{\partial t}+\left(\nabla \frac{\partial \psi_{D, t}^{k}}{\partial t}\right)^{T}\right): \nabla \psi_{D, t}^{i} d x-\int_{\partial D^{N}} \sigma\left(\frac{\partial \psi_{D, t}^{k}}{\partial t}\right) n \cdot \psi_{D, t}^{i} d S\right] \\
& =\frac{1}{\lambda_{D, t}^{k}}\left[-\eta \int_{D} \frac{\partial \psi_{D, t}^{k}}{\partial t} \cdot \Delta \psi_{D, t}^{i} d x+\eta \int_{\partial D} \frac{\partial \psi_{D, t}^{k}}{\partial t}\left(\nabla \psi_{D, t}^{i}+\left(\nabla \psi_{D, t}^{i}\right)^{T}\right) n d S\right.
\end{aligned}
$$




$$
\left.-\int_{\partial D^{N}} \sigma\left(\frac{\partial \psi_{D, t}^{k}}{\partial t}\right) n \cdot \psi_{D, t}^{i} d S\right] .
$$

Noting that

$$
\int_{D} \frac{\partial \psi_{D, t}^{k}}{\partial t} \cdot \nabla \phi_{D, t}^{i} d x=\int_{\partial D} \phi_{D, t}^{i} \frac{\partial \psi_{D, t}^{k}}{\partial t} \cdot n d S
$$

and using equation (5.16) once more, we get

$$
\begin{aligned}
\left(\frac{\partial \psi_{t}^{k}}{\partial t}, \psi_{t}^{i}\right)_{D, t}= & \frac{1}{\lambda_{D, t}^{k}}\left[\int_{D} \lambda_{D, t}^{i} \frac{\partial \psi_{D, t}^{k}}{\partial t} \cdot \psi_{D, t}^{i} d x-\int_{\partial D^{D}} \nu \frac{\partial \psi_{D, t}^{k}}{\partial n} \cdot \sigma\left(\psi_{D, t}^{i}\right) n d S\right. \\
& \left.-\int_{\partial D^{N}}\left(-\nu n \nabla \sigma\left(\psi_{D, t}^{k}\right) n-\sigma\left(\psi_{D, t}^{k}\right) \nabla_{\partial D} \nu\right) \cdot \psi_{D, t}^{i} d S\right] .
\end{aligned}
$$

Integrating by parts and doing some further simplification, we get (recall that $i \neq k$ ),

$$
\begin{aligned}
\left(\frac{\partial \psi_{D, t}^{k}}{\partial t}, \psi_{D, t}^{i}\right)_{D, t}= & \frac{-1}{\lambda_{D, t}^{k}-\lambda_{D, t}^{i}}\left[\int_{\partial D^{D}} \nu \sigma\left(\psi_{D, t}^{i}\right) n \cdot \frac{\partial \psi_{D, t}^{k}}{\partial n} d S\right. \\
& \left.+\int_{\partial D^{N}}\left(-\nu n \nabla \sigma\left(\psi_{D, t}^{k}\right) n+\sigma\left(\psi_{D, t}^{k}\right) \nabla_{\partial D} \nu\right) \cdot \psi_{D, t}^{i} d S\right] \\
= & \frac{-1}{\lambda_{D, t}^{k}-\lambda_{D, t}^{i}}\left[\int_{\partial D^{D}} \nu \sigma\left(\psi_{D, t}^{i}\right) n \cdot \frac{\partial \psi_{D, t}^{k}}{\partial n} d S\right. \\
& +\int_{\partial D^{N}}\left(-\nu n \nabla \sigma\left(\psi_{D, t}^{k}\right) n+\nu \nabla_{\partial D} \cdot \sigma\left(\psi_{D, t}^{k}\right)\right) \cdot \psi_{D, t}^{i} d S \\
& \left.+\int_{\partial D^{N}} \nu \sigma\left(\psi_{D, t}^{k}\right): \nabla_{\partial D} \psi_{D, t}^{i} d S\right] .
\end{aligned}
$$

Noting that $\nabla=\nabla_{\partial D}+n \frac{\partial}{\partial n}$, we have

$$
\begin{aligned}
= & \frac{-1}{\lambda_{D, t}^{k}-\lambda_{D, t}^{i}}\left[\int_{\partial D^{D}} \nu \sigma\left(\psi_{D, t}^{i}\right) n \cdot \frac{\partial \psi_{D, t}^{k}}{\partial n} d S\right. \\
& +\int_{\partial D^{N}} \nu \lambda_{D, t}^{k} \psi_{D, t}^{k} \cdot \psi_{D, t}^{i} d S \\
& \left.-\int_{\partial D^{N}} \nu \sigma\left(\psi_{D, t}^{k}\right): \nabla_{\partial D} \psi_{D, t}^{i} d S\right] .
\end{aligned}
$$

When $i=k$, we have

$$
\left(\frac{\partial \psi_{D, t}^{k}}{\partial t}, \psi_{D, t}^{k}\right)_{t}=0
$$

from the normalization condition $\left\|\psi_{D, t}^{k}\right\|_{\left(L^{2}(D)\right)^{d}}=1$. Integrating by parts twice and using equation (5.3), we have

$$
\begin{aligned}
& \left(v \nabla \psi_{D, t}^{k}, \psi_{D, t}^{i}\right)_{D, t}=-\frac{1}{\lambda_{D, t}^{k}} \int_{D} v \nabla\left(\nabla \cdot \sigma\left(\psi_{D, t}^{k}\right)\right) \psi_{D, t}^{i} d x \\
& \quad=\frac{1}{\lambda_{D, t}^{k}} \int_{D}\left[\left(\psi_{D, t}^{i} \nabla \sigma\left(\psi_{D, t}^{k}\right)\right): \nabla v+v\left(\nabla \sigma\left(\psi_{D, t}^{k}\right)\right): \nabla \psi_{D, t}^{i}\right] d x-\frac{1}{\lambda_{D, t}^{k}} \int_{\partial D}\left(\nabla \sigma\left(\psi_{D, t}^{k}\right) v\right): \psi_{D, t}^{i} n d S .
\end{aligned}
$$


Now, integrating by parts and using the fact that $\nabla \cdot v=0$, we have

$$
\int_{D}\left(\psi_{D, t}^{i} \nabla \sigma\left(\psi_{D, t}^{k}\right)\right): \nabla v d x=-\int_{D}\left(\nabla v \sigma\left(\psi_{D, t}^{k}\right)\right):\left(\nabla \psi_{D, t}^{i}\right)^{T} d x+\int_{\partial D}\left(\nabla v \sigma\left(\psi_{D, t}^{k}\right)\right): \psi_{D, t}^{i} n d S .
$$

Furthermore, by the definition of $\sigma\left(\psi_{D, t}^{k}\right)$ and the fact that $\nabla \cdot \psi_{D, t}^{i}=0$,

$$
\begin{aligned}
& \int_{D} v\left(\nabla \sigma\left(\psi_{D, t}^{k}\right)\right): \nabla \psi_{D, t}^{i} d x=\int_{D}\left[-v \cdot \nabla \phi_{D, t}^{k} \nabla \cdot \psi_{D, t}^{i}+\eta\left(v \nabla^{2} \psi_{D, t}^{k}\right): \nabla \psi_{D, t}^{i}+\eta\left(v \nabla^{2} \psi_{D, t}^{k}\right):\left(\nabla \psi_{D, t}^{i}\right)^{T}\right] d x \\
& =\eta \int_{D}\left[\left(v \nabla^{2} \psi_{D, t}^{k}\right): \nabla \psi_{D, t}^{i}+\left(v \nabla^{2} \psi_{D, t}^{k}\right):\left(\nabla \psi_{D, t}^{i}\right)^{T}\right] d x .
\end{aligned}
$$

Integrating both terms by parts, we have

$$
\begin{aligned}
= & -\eta \int_{D}\left[\left(\nabla \psi_{D, t}^{k} \nabla v\right): \nabla \psi_{D, t}^{i}+\left(\nabla \psi_{D, t}^{k} v\right) \cdot \Delta \psi_{D, t}^{i}\right] d x+\eta \int_{\partial D}\left(\nabla \psi_{D, t}^{k} v\right) \cdot\left(\nabla \psi_{D, t}^{i} n\right) d S \\
& -\eta \int_{D}\left(\nabla \psi_{D, t}^{k} \nabla v\right): \nabla \psi_{D, t}^{i} d x+\eta \int_{\partial D}\left(\nabla \psi_{D, t}^{k} v\right) \cdot\left(n \nabla \psi_{D, t}^{i}\right) d S .
\end{aligned}
$$

Using equation (5.3) and integrating by parts, we have

$$
\begin{aligned}
& \int_{D}\left(\nabla \psi_{D, t}^{k} v\right) \cdot \Delta \psi_{D, t}^{i} d x=\int_{D}\left[\lambda_{D, t}^{i}\left(\nabla \psi_{D, t}^{k} v\right) \cdot \psi_{D, t}^{i}-\left(\nabla \psi_{D, t}^{k} v\right) \cdot \nabla \phi_{D, t}^{i}\right] d x \\
& =\int_{D}\left[\lambda_{D, t}^{i}\left(\nabla \psi_{D, t}^{k} v\right) \cdot \psi_{D, t}^{i}+\nabla v:\left(\nabla \psi_{D, t}^{k}\right)^{T} \phi_{D, t}^{i}\right] d x-\int_{\partial D} n \nabla \psi_{D, t}^{k} v \phi_{D, t}^{i} d S
\end{aligned}
$$

Therefore, combining equations (5.20)-(5.23), we get

$$
\begin{aligned}
\left(v \nabla \psi_{D, t}^{k}, \psi_{D, t}^{i}\right)_{D, t}= & \frac{1}{\lambda_{D, t}^{k}-\lambda_{D, t}^{i}}\left[\int_{D}\left[-\left(\nabla v \sigma\left(\psi_{D, t}^{k}\right)\right):\left(\nabla \psi_{D, t}^{i}\right)^{T}-\left(\nabla \psi_{D, t}^{k} \nabla v\right): \sigma\left(\psi_{D, t}^{i}\right)\right] d x\right. \\
& +\int_{\partial D}\left[-\left(\nabla \sigma\left(\psi_{D, t}^{k}\right) v\right): \psi_{D, t}^{i} n+\left(\nabla v \sigma\left(\psi_{D, t}^{k}\right)\right): \psi_{D, t}^{i} n\right. \\
& \left.\left.+\left(\nabla \psi_{D, t}^{k} v\right) \cdot\left(\sigma\left(\psi_{D, t}^{i}\right) n\right)\right] d S\right] .
\end{aligned}
$$

Using the fact that $\nabla=\nabla_{\partial D}+n \frac{\partial}{\partial n}$, we can rewrite the boundary terms as

$$
\begin{aligned}
& \frac{1}{\lambda_{D, t}^{k}-\lambda_{D, t}^{i}}\left[\int_{\partial D}\left[-\left(\nabla \sigma\left(\psi_{D, t}^{k}\right) v\right): \psi_{D, t}^{i} n+\left(\nabla v \sigma\left(\psi_{D, t}^{k}\right)\right): \psi_{D, t}^{i} n+\left(\nabla \psi_{D, t}^{k} v\right) \cdot\left(\sigma\left(\psi_{D, t}^{i}\right) n\right)\right] d S\right] \\
&=\frac{1}{\lambda_{D, t}^{k}-\lambda_{D, t}^{i}}\left[\int_{\partial D^{D}} \nu \sigma\left(\psi_{D, t}^{i}\right) n \cdot \frac{\partial \psi_{D, t}^{k}}{\partial n} d S\right. \\
&\left.+\int_{\partial D^{N}} \nu \lambda_{D, t}^{k} \psi_{D, t}^{k} \cdot \psi_{D, t}^{i} d S-\int_{\partial D^{N}} \nu \sigma\left(\psi_{D, t}^{k}\right): \nabla_{\partial D} \psi_{D, t}^{i} d S\right] \\
&=\left(\frac{\partial \psi_{D, t}^{k}}{\partial t}, \psi_{D, t}^{i}\right) .
\end{aligned}
$$

In a similar fashion, using equation (5.3) and integrating by parts several times, we have

$$
\left(\nabla v \psi_{D, t}^{k}, \psi_{D, t}^{i}\right)_{D, t}=-\frac{1}{\lambda_{D, t}^{k}} \int_{D}\left(\psi_{D, t}^{i} \nabla v\right) \cdot \nabla \cdot \sigma\left(\psi_{D, t}^{k}\right) d x
$$




$$
=\frac{1}{\lambda_{D, t}^{k}} \int_{D}\left[\left(\psi_{D, t}^{i} \nabla^{2} v\right): \sigma\left(\psi_{D, t}^{k}\right)+\nabla v \sigma\left(\psi_{D, t}^{k}\right): \nabla \psi_{D, t}^{i}\right] d x
$$

From the definition of $\sigma_{D, t}$,

$$
\int_{D} \nabla v \sigma\left(\psi_{D, t}^{k}\right): \nabla \psi_{D, t}^{i} d x=\int_{D}\left[-\nabla v: \nabla \psi_{D, t}^{i} \phi_{D, t}^{k}+\eta \nabla v \nabla \psi_{D, t}^{k}: \nabla \psi_{D, t}^{i}+\eta \nabla v\left(\nabla \psi_{D, t}^{k}\right)^{T}: \nabla \psi_{D, t}^{i}\right] d x .
$$

Furthermore, integrating by parts and using equation (5.3), we have

$$
\begin{aligned}
\eta \int_{D} \nabla v \nabla \psi_{D, t}^{k}: \nabla \psi_{D, t}^{i}= & \eta \int_{D}\left[-\psi_{D, t}^{k} \nabla^{2} v: \nabla \psi_{D, t}^{i}+\nabla v \psi_{D, t}^{k} \cdot \Delta \psi_{D, t}^{i}\right] d x+\eta \int_{\partial D}\left(\nabla v \psi_{D, t}^{k}\right) \cdot\left(\nabla \psi_{D, t}^{i} \cdot n\right) d S \\
= & \int_{D}\left[-\eta \psi_{D, t}^{k} \nabla^{2} v: \nabla \psi_{D, t}^{i}+\nabla v \psi_{D, t}^{k} \cdot\left(\lambda_{D, t}^{i} \psi_{D, t}^{i}-\nabla \phi_{D, t}^{i}\right)\right] d x \\
& +\eta \int_{\partial D}\left(\nabla v \psi_{D, t}^{k}\right) \cdot\left(\nabla \psi_{D, t}^{i} \cdot n\right) d S .
\end{aligned}
$$

Integrating by parts once more and using equation (5.3), we have

$$
\int_{D} \nabla v \psi_{D, t}^{k} \cdot \nabla \phi_{D, t}^{i} d x=-\int_{D} \nabla v:\left(\nabla \psi_{D, t}^{k}\right)^{T} \phi_{D, t}^{i} d x+\int_{\partial D} \nabla v \psi_{D, t}^{k} \phi_{D, t}^{i} \cdot n d S .
$$

Therefore, combining equations (5.26)-(5.29), we get

$$
\begin{aligned}
\left(\nabla v \psi_{D, t}^{k}, \psi_{D, t}^{i}\right)_{D, t}= & \frac{1}{\lambda_{D, t}^{k}-\lambda_{D, t}^{i}}\left[\int _ { D } \left[\left(\psi_{D, t}^{i} \nabla^{2} v\right): \sigma\left(\psi_{D, t}^{k}\right)-\nabla v: \nabla \psi_{D, t}^{i} \phi_{D, t}^{k}+\eta \nabla v\left(\nabla \psi_{D, t}^{k}\right)^{T}: \nabla \psi_{D, t}^{i}\right.\right. \\
& \left.-\eta \psi_{D, t}^{k} \nabla^{2} v: \nabla \psi_{D, t}^{i}-\nabla v:\left(\psi_{D, t}^{k}\right)^{T} \phi_{D, t}^{i}\right] d x+\eta \int_{\partial D}\left(\nabla v \psi_{D, t}^{k}\right) \cdot\left(\nabla \psi_{D, t}^{i} \cdot n\right) d S \\
& \left.-\int_{\partial D} \nabla v \psi_{D, t}^{k} \phi_{D, t}^{i} \cdot n d S\right] .
\end{aligned}
$$

Adding

we get

$$
\int_{\partial D} \nabla \psi_{D, t}^{i} \nabla v \psi_{D, t}^{k} \cdot n d S=\int_{D}\left[\nabla^{2} v \psi_{D, t}^{k}:\left(\nabla \psi_{D, t}^{i}\right)^{T}+\nabla v \nabla \psi_{D, t}^{k}:\left(\nabla \psi_{D, t}^{i}\right)^{T}\right] d x
$$

$$
\begin{aligned}
\left(\nabla v \psi_{D, t}^{k}, \psi_{D, t}^{i}\right)_{D, t}= & \frac{1}{\lambda_{D, t}^{k}-\lambda_{D, t}^{i}}\left[\int _ { D } \left[\left(\psi_{D, t}^{i} \nabla^{2} v\right): \sigma\left(\psi_{D, t}^{k}\right)-\nabla v: \nabla \psi_{D, t}^{i} \phi_{D, t}^{k}+\nabla v\left(\nabla \psi_{D, t}^{k}\right)^{T}: \sigma\left(\psi_{D, t}^{i}\right)\right.\right. \\
& \left.\left.-2 \eta \psi_{D, t}^{k} \nabla^{2} v: e\left(\psi_{D, t}^{i}\right)\right] d x\right] .
\end{aligned}
$$

Combining equations (5.18), (5.19), (5.24), (5.25), and (5.30)), we get that, for $i \neq k$,

$$
\begin{aligned}
a_{i}^{k}= & \frac{1}{\lambda_{D, t}^{k}-\lambda_{D, t}^{i}}\left[\int _ { D } \left[-2 \eta\left(\nabla v e\left(\psi_{D, t}^{k}\right)\right):\left(\nabla \psi_{D, t}^{i}\right)^{T}-2\left(\nabla \psi_{D, t}^{k} e(v)\right): \sigma\left(\psi_{D, t}^{i}\right)-\left(\psi_{D, t}^{i} \nabla^{2} v\right): \sigma\left(\psi_{D, t}^{k}\right)\right.\right. \\
& \left.\left.+2 e(v): \nabla \psi_{D, t}^{i} \phi_{D, t}^{k}+2 \eta \psi_{D, t}^{k} \nabla^{2} v: e\left(\psi_{D, t}^{i}\right)\right] d x\right],
\end{aligned}
$$

which, integrating by parts and simplifying, we can write

$$
=\frac{1}{\lambda_{D, t}^{k}-\lambda_{D, t}^{i}}\left[-2 \int_{D} \nabla \phi_{D, t}^{k} e(v) \cdot \psi_{D, t}^{i} d x+2 \eta \int_{D}\left(\psi_{D, t}^{i} \nabla^{2} \psi_{D, t}^{k}\right): e(v) d x+\int_{D}\left(\nabla \psi_{D, t}^{k} \Delta v\right) \cdot \psi_{D, t}^{i} d x\right.
$$




$$
\begin{aligned}
& +\eta \int_{D}\left(\nabla^{2} v \psi_{D, t}^{i}\right): \nabla \psi_{D, t}^{k} d x-2 \eta \int_{D} \psi_{D, t}^{i} \nabla^{2} v: \nabla \psi_{D, t}^{k} d x-\eta \int_{D} \psi_{D, t}^{i} \nabla \Delta v \cdot \psi_{D, t}^{k} d x \\
& \left.-\eta \int_{D}\left(\nabla^{2} v \psi_{D, t}^{i}\right): \nabla \psi_{D, t}^{k} d x\right] .
\end{aligned}
$$

For $i=k$,

$$
a_{k}^{k}=-\int_{D(0)}\left(\psi_{D, 0}^{k} e(v)\right) \cdot \psi_{D, 0}^{k} d x
$$

From equations (5.7), (4.1), and (5.11), $\mathcal{A}_{\tau}=1+\mathcal{O}(\tau)$. Therefore, $\exists C>0$ such that

$$
\begin{aligned}
\left\|\psi_{D, t_{n}}^{k}-\mathcal{A}_{D, t_{n}} \psi_{D, 0}^{k}\right\|_{\left(H^{1}\left(D\left(t_{n}\right)\right)\right)^{d}} & \leq \sum_{i=1}^{n}\left\|\mathcal{A}_{(n-i) \tau}\left(\psi_{D, t_{i}}^{k}-\mathcal{A}_{\tau} \psi_{D, t_{i-1}}^{k}\right)\right\|_{\left(H^{1}\left(D\left(t_{n}\right)\right)\right)^{d}} \\
& \leq \sum_{i=1}^{n}\left\|\psi_{D, t_{i}}^{k}-\mathcal{A}_{\tau} \psi_{D, t_{i-1}}^{k}\right\|_{\left(H^{1}\left(D\left(t_{i}\right)\right)\right)^{d}}+C \tau^{2} .
\end{aligned}
$$

Therefore, noting equations (5.7), (5.13), (5.15), (5.31), and (5.32), we have that $\exists C>0$ such that

$$
\begin{aligned}
& \left\|\psi_{D, t_{n}}^{k}-\mathcal{A}_{t_{n}} \psi_{D, 0}^{k}\right\|_{\left(L^{2}(D(0))\right)^{d}} \leq \tau \sum_{i=1}^{N} \sqrt{\sum_{j=M+1}^{\infty}\left(a_{j}^{k}\left(t_{i}\right)\right)^{2}}+C \tau^{2} \\
& \quad \leq \tau \sum_{i=1}^{N} \frac{1}{\left|\lambda_{D, t_{i}}^{k}-\lambda_{D, t_{i}}^{M+1}\right|}\left[2 \beta \lambda_{D, t_{i}}^{k}\left\|e\left(v_{t_{i}}\right)\right\|_{L^{\infty}}+4 \sqrt{\lambda_{D, t_{i}}^{k}}\left\|\nabla^{2} v_{t_{i}}\right\|_{L^{\infty}}\right]+C \tau^{2},
\end{aligned}
$$

where $\beta$ is defined in equation (5.9). Similarly,

$$
\begin{aligned}
& \left\|\nabla \psi_{D, t_{n}}^{k}-\nabla \mathcal{A}_{t_{n}} \psi_{D, 0}^{k}\right\|_{\left(L^{2}(D(0))\right)^{d}} \leq \tau \sqrt{\sum_{i=M+1}^{\infty} \lambda_{D, t_{n}}^{i}\left(a_{i}^{k}\right)^{2}}+C \tau^{2} \\
& \quad \leq \tau \sum_{i=1}^{N} \frac{\sqrt{\lambda_{D, t_{i}}^{M+1}}}{\left|\lambda_{D, t_{i}}^{k}-\lambda_{D, t_{i}}^{M+1}\right|}\left[2 \beta \lambda_{D, t_{i}}^{k}\left\|e\left(v_{t_{i}}\right)\right\|_{L^{\infty}}+4 \sqrt{\lambda_{D, t_{i}}^{k}}\left\|\nabla^{2} v_{t_{i}}\right\|_{L^{\infty}}\right]+C \tau^{2} .
\end{aligned}
$$

Now, from the max-min property of eigenvalues (see, e.g., [3], the eigenvalues $\lambda_{D, t}^{k}$ are bounded above by the Dirichlet eigenvalues and below by Neumann eigenvalues. From the results in [29], these eigenvalues, and hence $\lambda_{D, t}^{k}$, have the same asymptotic behavior. Therefore, as $l \rightarrow \infty$,

$$
\lambda_{D, t}^{l} \sim \eta\left(\frac{(2 \pi)^{d}}{\omega_{d}(d-1)}\right)^{\frac{2}{d}}|D|^{-2 / d} l^{2 / d} .
$$

Now, taking the limits $h \rightarrow 0$ and $\tau \rightarrow 0$ in equations (5.33) and (5.34), we get the desired result.

Let $V^{h}(D):=\operatorname{span}\left(\left\{\psi_{D, t}^{k}\right\}\right)$. The full error estimate in $D$ is given by the following theorem.

Theorem 5.3. Let $u$ solve equation (2.2), and let $u_{D, h}(T)=\sum_{k=1}^{M} a_{k} \psi_{T}^{k}$, where $M:=h^{-d}|D|$, be the Galerkin solution of equation (2.2) in $V^{h}(D(T))$. Let $\tilde{u}_{D, h}:=\sum_{k=1}^{M} a_{k} \mathcal{A}_{T} \psi_{D, 0}^{k}$. Then

$$
\begin{aligned}
\| u(T) & -\hat{u}_{D, h}(T) \|_{\left(H^{1}(D(T))\right)^{d}} \\
& \leq\left\|u(T)-\tilde{u}_{D, h}(T)\right\|_{\left(H^{1}(D(T))\right)^{d}}
\end{aligned}
$$




$$
\leq\left\|u(T)-u_{D, h}(T)\right\|_{\left(H^{1}(D(T))\right)^{d}}+\left\|u_{D, h}(T)-\tilde{u}_{D, h}(T)\right\|_{\left(H^{1}(D(T))\right)^{d}},
$$

where

$$
\lim _{h \rightarrow 0} \frac{\left\|u(T)-u_{D, h}(T)\right\|_{\left(H^{1}(D(T))\right)^{d}}}{h\|f\|_{\left(L^{2}(D(T))\right)^{d}}}=\left(\frac{\omega_{d}(d-1)}{(2 \pi)^{d}}\right)^{\frac{1}{d}}
$$

and

$$
\begin{aligned}
& \lim _{\tau \rightarrow 0} \lim _{h \rightarrow 0} \frac{\left\|u_{D, h}(T)-\tilde{u}_{D, h}(T)\right\|_{\left(H^{1}(D(T))\right)^{d}}}{h\|f\|_{\left(H^{(d+1) / 2}(D(T))\right)^{d}}} \\
& \leq \frac{2 \beta d\left|V_{F}\right|^{1 / d}}{(2 \pi)^{1 / 2+d} \eta^{1 / 4+d}\left((d-1)\left|V_{F}\right| \omega_{d}\right)^{1+1 / 2 d}} \int_{0}^{T}\|e(v(t))\|_{\left(L^{\infty}\left(V_{F}(t)\right)\right)^{d \times d}} d t
\end{aligned}
$$

Proof. Equation (5.36) follows from the triangle inequality and the definition of $\hat{u}_{D, h}$. Equation (5.37) follows from an argument identical to that used to derive equation (3.4). Note that

$$
\begin{aligned}
\left\|u_{D, h}\left(t_{n}\right)-\tilde{u}_{D, h}\left(t_{n}\right)\right\|_{\left(H^{1}\left(D\left(t_{n}\right)\right)\right)^{d}} & =\left\|\sum_{k=1}^{M} a_{k}\left(\psi_{D, t_{n}}^{k}-\mathcal{A}_{t_{n}} \psi_{D, 0}^{k}\right)\right\|_{\left(H^{1}\left(D\left(t_{n}\right)\right)\right)^{d}} \\
& =\left\|\sum_{k=1}^{M}\left(\lambda_{D, t_{n}}^{k}\right)^{1+\alpha / 2} a_{k} \frac{\psi_{D, t_{n}}^{k}-\mathcal{A}_{t_{n}} \psi_{D, 0}^{k}}{\left(\lambda_{D, t_{n}}^{k}\right)^{1+\alpha / 2}}\right\|_{\left(H^{1}\left(D\left(t_{n}\right)\right)\right)^{d}} \\
& \leq\left(\sum_{k=1}^{M}\left(\lambda_{D, t_{n}}^{k}\right)^{2+\alpha} a_{k}^{2}\right)^{\frac{1}{2}}\left(\sum_{k=1}^{M}\left\|\frac{\psi_{D, t_{n}}^{k}-\mathcal{A}_{t_{n}} \psi_{D, 0}^{k}}{\left(\lambda_{D, t_{n}}^{k}\right)^{1+\alpha / 2}}\right\|_{\left(H^{1}\left(D\left(t_{n}\right)\right)\right)^{d}}^{2}\right)^{\frac{1}{2}} \\
& \leq\|f\|_{\left(H^{\alpha}\left(D\left(t_{n}\right)\right)\right)^{d}}\left(\sum_{k=1}^{M} \frac{\left\|\psi_{D, t_{n}}^{k}-\mathcal{A}_{t_{n}} \psi_{D, 0}^{k}\right\|_{\left(H^{1}\left(D\left(t_{n}\right)\right)\right)^{d}}^{2}}{\left(\lambda_{D, t_{n}}^{k}\right)^{2+\alpha}} .\right.
\end{aligned}
$$

Setting $\alpha=\frac{2 d-1}{2}$, applying Theorem 5.2, equation (5.35), and taking the limit $\tau \rightarrow 0$, we get equation (5.38).

\section{Conclusion}

In this report we have considered the problem of efficient numerical upscaling of a Stokesian particle system with a fine-grained evolving geometry. The main result follows by constructing a provably optimal (albeit impractical) spectral discretization basis, capturing the system down to a given scale. Because of its spectral nature, an exact evolution law for the basis follows from the analog of Hadamard's formula for the change in the eigenfunctions due to an infinitesimal perturbation in the domain. The basis evolution law allows us to design an upscaled basis update procedure in response to the movement of the domain, while maintaining full error control. We propose a particular update procedure, but its numerical assessment as well as the search for, possibly, better update methods is outside the scope of this report. Further, we propose a variation of the basis localization technique to convert the spectral basis into a practical discretization scheme.

\section{Acknowledgments}

This work was done during the first author's stay at Argonne National Laboratory in the summer of 2010 as a Givens Fellow in the Mathematics and Computer Science Division; he expresses his gratitude for the 
hospitality during his visit. The authors also express their gratitude to Igor Aronson for insightful discussions on the problem of numerical treatment of Stokesian suspensions. They are further indebted to Lei Zhang and Houman Owhadi for making an early draft of their basis localization paper available. This work was

supported in part by the Office of Advanced Scientific Computing Research, Office of Science, U.S. Dept. of Energy, under Contract DE-AC02-06CH11357. The work of BMH was supported in part by the DOE grant DE-FG02-08ER25862. The work of LVB was supported by DOE grant DE-FG02-08ER25862.

\section{References}

[1] K. I. Babenko. On the asymptotic behavior of the eigenvalues of linearized navier-stokes equations. Dokl. Akad. Nauk SSSR, 263:521-525, 1982.

[2] L. H. Cisneros, R. Cortez, C. Dombrowski, R. E. Goldstein, and J. O. Kessler. Fluid dynamics of selfpropelled migroorganisms, from individuals to concentrated populations. Experimental Fluids, 43:737$753,2007$.

[3] R. Courant and D. Hilbert. Methods of Mathematical Physics. Intercience Publishers, Inc., 1965.

[4] A. Czirók, H. E. Stenley, and T. Vicsek. Spontaneously ordered motion of self-propelled particles. J. Phys. A: Math. Gen., 30:1375, 1997.

[5] C. Dombrowski, L. Cisneros, S. Chatkaew, R. E. Goldstein, and J. O. Kessler. Self-concentration and large-scale coherence in bacterial dynamics. Phys. Rev. Lett., 93:098103, 2004.

[6] T. Geveci, B. D. Reddy, and H. T. Pearce. On the approximation of the spectrum of the stokes operators. Mathematical Modelling and Numerical Analysis, 23(1):129-136, 1989.

[7] G. Grégoire and H. Chaté. Onset of collective and cohesive motion. Phys. Rev. Lett., 92:025702, 2004.

[8] P. Grinfeld. Hadamard's formula inside and out. J. Optim. Theory Appl., 146:654-690, 2010.

[9] M. Günther and G. Prokert. Existence results for the quasistationary motion of a capillary liquid drop. Zeitschrift für Analysis, 16(2):311-348, 1997.

[10] B. M. Haines, I. S. Aranson, L. Berlyand, and D. A. Karpeev. Effective viscosity of dilute bacterial suspensions: a two-dimensional model. Phys. Biol., 5:046003, 2008.

[11] B. M. Haines, I. S. Aranson, L. Berlyand, and D. A. Karpeev. Effective viscosity of bacterial suspensions: a three-dimensional PDE model with stochastic torque. Comm. Pure Appl. Anal., 11:19-46, 2012.

[12] B. M. Haines, A. Sokolov, I. S. Aranson, L. Berlyand, and D. A. Karpeev. A three-dimensional model for the effective viscosity of bacterial suspensions. Phys. Rev. E, 80:041922, 2009.

[13] Y. Hatwalne, S. Ramaswamy, M. Rao, and R. A. Simha. Rheology of active-particle suspensions. Phys. Rev. Lett., 92:118101, 2004.

[14] J. P. Hernandez-Ortiz, C. G. Stoltz, and M. D. Graham. Transport and collective dynamics in suspensions of confined swimming particles. Phys. Rev. Lett., 95:204501, 2005.

[15] M. Ibele, T. Mallouk, and A. Sen. Schooling behavior of light-powered autonomous micromoters in water. Angewandte Chemie International Edition, 48:3308-3312, 2009.

[16] T. Ishikawa and T. J. Pedley. The rheology of a semi-dilute suspension of swimming model microorganisms. J. Fluid Mech., 588:399, 2007.

[17] T. Ishikawa and T. J. Pedley. Coherent structures in monolayers of swimming particles. Phys. Rev. Lett., 100:088103, 2008. 
[18] M. J. Kim and K. S. Breuer. Enhanced diffusion due to motile bacteria. Phys. Fluids, 16(9):L78-L81, 2004.

[19] M. J. Kim and K. S. Breuer. Use of bacterial carpets to enhance mixing in microfluidic systems. Jour. Fluids Engin., 129(319), 2007.

[20] K. C. Leptos, J. S. Guasto, J. P. Gollub, A. I. Pesci, and R. E. Goldstein. Dynamics of enhanced tracer diffusion in suspensions of swimming eukaryotic microorganisms. Phys. Rev. Lett., 103(19):198103, 2009.

[21] I. Llopis and I. Pagonabarraga. Dynamic regimes of hydronamically coupled self-propelling particles. Europhys. Lett., 75:999-1005, 2006.

[22] N. H. Mendelson, A. Bourque, K. Wilkening, K. R. Anderson, and J. C. Watkins. Organized cell swimming motions in Bacillus subtilis colonies: Patterns of short-lived whirls and jets. J. Bacteriol., 181:600-609, 1999.

[23] B. Mercier, J. Osborn, J. Rappaz, and P. A. Raviart. Eigenvalue approximations by mixed and hybrid methods. Mathematics of Computation, 36(154):427-453, 1981.

[24] G. Métivier. Valeurs propres des opérateurs définis par la restriction de systèmes variationnels a des sous-espases. J. Math. Pures Appl., 57:133-156, 1978.

[25] T. J. Pedley and J. O. Kessler. A new continuum model for suspensions of gyrotactic micro-organisms. J. Fluid Mech., 212:155-182, 1990.

[26] S. Rafaï, L. Jibuti, and P. Peyla. Effective viscosity of microswimmer suspensions. Phys. Rev. Lett., 104:098102, 2010.

[27] I. H. Riedel, K. Kruse, and J. Howard. A self-organized vortex array of hydrodynamically entrained sperm cells. Science, 309(5732):300-303, 2005.

[28] S. D. Ryan, B. M. Haines, L. Berlyand, F. Ziebert, and I. S. Aranson. Viscosity of bacterial suspensions: Hydrodynamic interactions and self-induced noise. Phys. Rev. E, 83:050904(R), 2011.

[29] Y. Safarov. On the comparison of the dirichlet and neumann counting functions. In T. Suslina and D. Yafaev, editors, American Mathematical Society Translations Series 2: Spectral theory of differential operators, volume 225, pages 191-204. American Mathematical Society, Providence, RI, 2008.

[30] D. Saintillan. The dilute rheology of swimming suspensions: A simple kinetic model. Experimental Mechanics, 50:1275-1281, 2010.

[31] D. Saintillan. Extensional rheology of active suspensions. Phys. Rev. E, 81:056307, 2010.

[32] D. Saintillan and M. J. Shelley. Orientational order and intabilities in suspensions of self-locomoting rods. Phys. Rev. Lett., 99:058102, 2007.

[33] A. Sokolov and I. S. Aranson. Reduction of viscosity in suspension of swimming bacteria. Phys. Rev. Lett., 103(14):148101, 2009.

[34] A. Sokolov, I. S. Aranson, J. O. Kessler, and R. E. Goldstein. Concentration dependence of the collective dynamics of swimming bacteria. Phys. Rev. Lett., 98:158102, 2007.

[35] A. Sokolov, R. E. Goldstein, F. I. Feldchtein, and I. S. Aranson. Enhanced mixing and spatial instability in concentrated bacterial suspensions. Phys. Rev. E, 80:031903, 2009.

[36] R. Temam. Navier-Stokes Equations and Nonlinear Functional Analysis. SIAM, 1995.

[37] T. Thomas. Extended compatibility conditions for the study of surface discontinuity in continuum mechanics. J. Math. Mech., 6:311-322, 1957. 
[38] I. Tuval, L. Cisneros, C. Dombrowski, C. W. Wolgemuth, J. O. Kessler, and R. E. Goldstein. Bacterial swimming and oxygen transport near contact lines. PNAS, 102(7):2277-2282, 2005.

[39] P. T. Underhill, J. P. Hernandez-Ortiz, and M. D. Graham. Diffusion and spatial correlations in suspensions of swimming particles. Phys. Rev. Lett., 100:248101, 2008.

[40] M. Wu, J. W. Roberts, S. Kim, D. L. Koch, and M. P. Delisa. Collective bacterial dynamics revealed using a three-dimensional population-scale defocused particle tracking technique. Applied and Environmental Microbiology, 72(7):4987-4994, 2006.

[41] X.-L. Wu and A. Libchaber. Particle diffusion in a quasi-two-dimensional bacterial bath. Phys. Rev. Lett., 84:3017, 2000.

[42] H. P. Zhang, Avraham Be'er, E.-L. Florin, and Harry L. Swinney. Collective motion and density fluctuations in bacterial colonies. PNAS, 107(31):13626-13630, 2010. 
Argonne

Mathematics and Computer Science Division

Argonne National Laboratory

9700 South Cass Avenue, BIdg. 240

Argonne, IL 60439-4847

www.anl.gov 